\title{
Measurements of the $\mathrm{pp} \rightarrow \mathrm{W}^{ \pm} \gamma \gamma$ and $\mathrm{pp} \rightarrow \mathrm{Z}_{\gamma \gamma}$ cross sections at $\sqrt{\mathrm{s}}=13 \mathrm{TeV}$ and limits on anomalous quartic gauge couplings
}

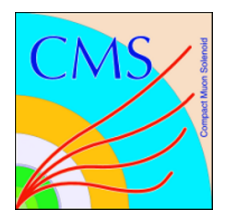

\section{The CMS collaboration}

E-mail: cms-publication-committee-chair@cern.ch

ABSTRACT: The cross section for $\mathrm{W}$ or $\mathrm{Z}$ boson production in association with two photons is measured in proton-proton collisions at a centre-of-mass energy of $13 \mathrm{TeV}$. The data set corresponds to an integrated luminosity of $137 \mathrm{fb}^{-1}$ collected by the CMS experiment at the LHC. The $\mathrm{W} \rightarrow \ell v$ and $\mathrm{Z} \rightarrow \ell \ell$ decay modes (where $\ell=\mathrm{e}, \mu$ ) are used to extract the $\mathrm{W} \gamma \gamma$ and $\mathrm{Z} \gamma \gamma$ cross sections in a phase space defined by electron (muon) with transverse momentum larger than $30 \mathrm{GeV}$ and photon transverse momentum larger than $20 \mathrm{GeV}$. All leptons and photons are required to have absolute pseudorapidity smaller than 2.5. The measured cross sections in this phase space are $\sigma(\mathrm{W} \gamma \gamma)=13.6_{-1.9}^{+1.9}$ (stat) $)_{-4.0}^{+4.0}$ (syst) \pm $0.08(\mathrm{PDF}+\mathrm{scale}) \mathrm{fb}$ and $\sigma(\mathrm{Z} \gamma \gamma)=5.41_{-0.55}^{+0.58}$ (stat) $)_{-0.70}^{+0.64}$ (syst) $\pm 0.06(\mathrm{PDF}+$ scale $) \mathrm{fb}$. Limits on anomalous quartic gauge couplings are set in the framework of an effective field theory with dimension-8 operators.

KEYWORDS: Hadron-Hadron scattering (experiments), Particle and resonance production ArXiv EPRINT: 2105.12780 


\section{Contents}

1 Introduction 1

2 The CMS detector 2

3 Event simulation 3

4 Event selection $\quad 4$

$\begin{array}{lll}5 & \text { Background estimation } & 6\end{array}$

$\begin{array}{lll}6 & \text { Systematic uncertainties } & 7\end{array}$

$\begin{array}{lll}7 & \text { Cross section measurements } & 10\end{array}$

8 Limits on anomalous quartic gauge couplings $\quad 13$

9 Summary 14

$\begin{array}{lr}\text { The CMS collaboration } & 19\end{array}$

\section{Introduction}

The measurement of the associated production of a vector boson $\mathrm{V}(=\mathrm{W}, \mathrm{Z})$ and two photons in proton-proton (pp) collisions is a powerful test of the standard model (SM). The nonabelian nature of the electroweak interaction predicts the presence of self-interacting vector boson vertices. The strength of the interaction is set by the values of triple and quartic gauge couplings predicted by the SM. The measurement of possible deviations from the theoretical predictions could provide indirect evidence of new particles or new interactions. Discrepancies at high photon momentum, where new physics might give a measurable deviation from the SM cross section, would produce evidence for the possible existence of anomalous quartic gauge couplings (aQGCs). A parametrisation of predictions involving anomalous couplings, independent of any specific new physics model, can be calculated in an effective field theory (EFT) framework [1]. Triboson production is also an important background for several SM and beyond the SM processes, such as the Higgs boson production in association with vector bosons (with $\mathrm{H} \rightarrow \gamma \gamma$ ). Thus, studies of the self-couplings of the electroweak gauge bosons provide an excellent opportunity for a deeper understanding of electroweak interactions.

Some of the elementary processes resulting in the production of a massive vector boson in association with two photons at the CERN LHC are presented in the leading-order (LO) Feynman diagrams of figure 1. Topologies with final states that originate from events where 

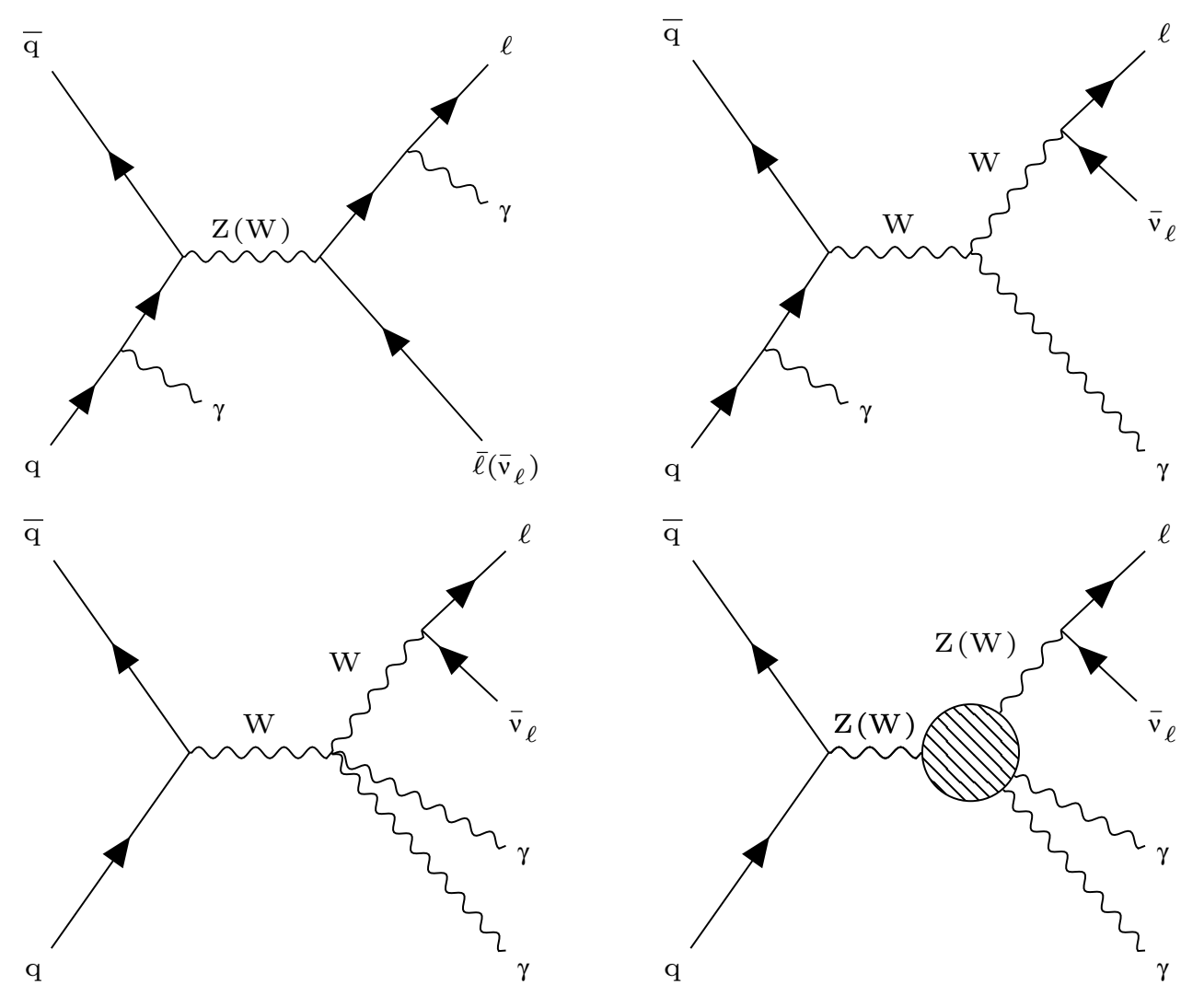

Figure 1. Representative Feynman diagrams for the $\mathrm{V} \gamma \gamma$ production in the SM (top left, top right and bottom left) and beyond the SM (bottom right).

the $\mathrm{V}$ boson is produced in the hard interaction between the two partons, and the photons come from either initial or final state radiation processes or from quartic gauge vertices together with the $\mathrm{V}$ boson, are studied, and are referred to as $\mathrm{V} \gamma \gamma$ in the text.

Previous measurements of the $\mathrm{V} \gamma \gamma$ production cross sections have been performed by the ATLAS and CMS collaborations at the CERN LHC in pp collisions at a centre-of-mass energy of $\sqrt{s}=8 \mathrm{TeV}[2-4]$. Limits on the presence of aQGCs were also reported in these papers.

In this paper, the first measurements of the $\mathrm{pp} \rightarrow \mathrm{W} \gamma \gamma$ and $\mathrm{pp} \rightarrow \mathrm{Z} \gamma \gamma$ cross sections at $\sqrt{s}=13 \mathrm{TeV}$ are presented using data collected between 2016 and 2018 by the CMS experiment, corresponding to an integrated luminosity of $137 \mathrm{fb}^{-1}$. For these measurements, only direct decays into electrons or muons are considered. Measurements are compared with the latest available calculations at next-to-LO (NLO) in perturbative quantum chromodynamics (QCD) [5-7]. Limits on the aQGCs are presented in the framework of an electroweak EFT with dimension-8 operators.

\section{The CMS detector}

The central feature of the CMS apparatus is a superconducting solenoid of $6 \mathrm{~m}$ internal diameter, providing a magnetic field of $3.8 \mathrm{~T}$. Within the solenoid volume are a silicon 
pixel and strip tracker, a lead tungstate crystal electromagnetic calorimeter (ECAL), and a brass and scintillator hadron calorimeter (HCAL), each composed of a barrel and two endcap sections. Forward calorimeters extend the pseudorapidity $\eta$ coverage provided by the barrel and endcap detectors. Muons are detected in gas-ionisation chambers embedded in the steel flux-return yoke outside the solenoid.

Events of interest are selected using a two-tiered trigger system [8]. The first level, composed of custom hardware processors, uses information from the calorimeters and muon detectors to select events at a rate of around $100 \mathrm{kHz}$ with a latency of about $4 \mu \mathrm{s}$. The second level, known as the high-level trigger, consists of a farm of processors running a version of the full event reconstruction software optimised for fast processing that reduces the event rate to around $1 \mathrm{kHz}$ before data storage.

A more detailed description of the CMS detector, together with a definition of the coordinate system used and the relevant kinematic variables, is reported in ref. [9].

\section{Event simulation}

The associated production of a $\mathrm{W}(\mathrm{Z})$ boson and at least two photons is searched for in events with one lepton (two opposite-sign, same-flavour leptons) and two photons. Only electron and muon decay channels are used, while the $\tau$ decays are treated as a background. The $\mathrm{V} \gamma \gamma$ signal samples are generated at NLO with MADGRAPH5_amC@NLO [10] (2.2.2 for 2016 samples, 2.2 .6 for 2017 and 2018 samples) with no additional jets in the matrix element calculation. The NLO NNPDF 3.0 set [11] (for 2016 samples) and the next-to-NLO NNPDF 3.1 set [12] (for 2017 and 2018 samples) are used as parton distribution function (PDF) sets.

The main background contribution comes from the misidentification of jets as photons, which is estimated in single-photon control regions. Thus, various background samples involving a single photon are needed, including $\mathrm{V}(\mathrm{V}) \gamma$ samples. Other single-photon and diphoton processes (such as t $\bar{t}$ produced in association with one or two photons or a photon and a jet) contribute as backgrounds and are estimated using Monte Carlo (MC) simulations. The background contribution from the associated production of a $\mathrm{W}(\mathrm{Z})$ boson and a Higgs boson decaying to two photons is negligible, and is not considered.

The $\mathrm{V} \gamma$, the $\mathrm{t} \gamma$ and the $\mathrm{t} \overline{\mathrm{t}} \gamma$ samples are generated at NLO with MADGRAPH5 aMC@NLO with up to one additional jet in the matrix element calculation. The VV $\gamma$ and the $t \overline{\mathrm{t}} \gamma \gamma$ samples are generated at NLO with MADGRAPH5_aMC@NLO with no additional jets in the matrix element calculation. The $\gamma$ plus jets samples are generated at LO with MADGraph5_amC@NLO. The same PDF sets as for the signal samples are used. Alternative $\mathrm{V} \gamma$ samples, which are generated with SHERPA v2.2.6 [13, 14] at NLO precision with up to two additional jets and at LO precision for the three-jet computation using the NNPDF 3.1 set, are used for consistency checks and systematic uncertainties evaluations.

The PYTHIA v.8.226 (v.8.230) package version is used for hadronisation with the CUETP8M1 tune [15] (CP5 tune [16]) for the 2016 (2017 and 2018) samples.

Photons can be present also in other processes because of the hadronisation phase of the generation performed with PYTHIA (v.8.2) even if not explicitly produced at matrix 
element level. To avoid possible double counting effects in the event selection, a procedure for the removal of the overlapping phase space region between inclusive and exclusive samples is implemented. Photons are selected at the generator level following a selection as close as possible to the one performed at reconstruction level. The total number of selected photons at the generator level is then used to remove the overlapping phase space between different samples. For single-photon processes (such as $\mathrm{W} \gamma$ or $\mathrm{Z} \gamma$ ), the event is discarded if the total number of selected photons at the generator level is different from one. The event is discarded from the diphoton processes if it has less than two photons selected at the generator level.

Predictions for aQGC signals are obtained by including a set of weights, corresponding to the presence of the anomalous couplings, to the $\mathrm{V} \gamma \gamma$ reference samples simulated with MAdGraPh5_amC@NLO. For this purpose, an aQGC model [17] is used.

Additional pp interactions in the same or adjacent bunch crossings (known as pileup) is included by adding simulated minimum bias events to the hard scattering. The events in the MC simulations are weighted so the distribution of the number of pileup interactions matches the one measured in data. The interaction of the particles with the CMS detector is simulated with GEANT4 [18].

\section{Event selection}

Events for the $\mathrm{V} \gamma \gamma$ analysis are selected using isolated single-lepton trigger requirements [8]. Single-electron trigger algorithms have a transverse-momentum $p_{\mathrm{T}}$ threshold of $27 \mathrm{GeV}$ (for the 2016 data-taking period) and $32 \mathrm{GeV}$ (for the 2017 and 2018 data-taking periods); single-muon trigger algorithms require $p_{\mathrm{T}}$ above $24 \mathrm{GeV}$ for all three years.

All measured particles are reconstructed using the particle-flow (PF) algorithm [19]; this algorithm reconstructs and identifies each individual particle in an event with an optimised combination of information from the various elements of the CMS detector. The reconstructed vertex with the largest value of the sum of the $p_{\mathrm{T}}^{2}$ of the physics objects is the primary pp interaction vertex. The photon energies are obtained from the ECAL measurement. The electron energies are determined from a combination of the electron momentum at the primary interaction vertex as determined by the tracker, the energy of the corresponding ECAL cluster, and the energy sum of all bremsstrahlung photons spatially compatible with the ones originating from the electron track. The muon energies are obtained from the curvature of the muon tracks.

Electrons candidates are required to have $p_{\mathrm{T}}>15 \mathrm{GeV}$ in the pseudorapidity ranges that exclude the barrel-endcap transition region, $|\eta|<1.44$ and $1.57<|\eta|<2.50$. A variety of criteria is used to separate genuine electrons from misidentified ones. A tight identification is used to select prompt electrons (produced at the primary vertex) and isolated electrons in the final state [20]. Background contributions from misidentified jets or electrons inside a jet are rejected applying electron isolation criteria, which exploit the PF-based event reconstruction. The electron isolation variables are obtained by summing the $p_{\mathrm{T}}$ of charged hadrons compatible with the primary vertex $I_{\mathrm{chg}}$, of neutral hadrons $I_{\text {neu }}$, and of photons $I_{\text {pho }}$ inside a cone of radius $\Delta R=\sqrt{(\Delta \eta)^{2}+(\Delta \phi)^{2}}=0.3$ around 
the electron direction, where $\phi$ is the azimuthal angle in radians. Additional photons and neutral hadronic contributions to the isolation variable, coming from pileup, are subtracted using the jet area approach [21].

Muons candidates are required to have $p_{\mathrm{T}}>15 \mathrm{GeV}$ in the pseudorapidity range $|\eta|<2.4$. Muon identification criteria are based on the fit quality for tracks measured in the tracker and muon detectors. A tight muon identification is used to reconstruct muons in the final state [22]. To distinguish between prompt muons and those from hadron decays within jets, muons are required to be isolated with respect to all nearby $\mathrm{PF}$ reconstructed particles. For the computation of the PF isolation, the $I_{\text {chg }}, I_{\text {neu }}$ and $I_{\text {pho }}$ components are summed in a cone of $\Delta R=0.4$ around the muon direction. The corrected energy sum is obtained by subtracting the pileup contribution to $I_{\text {neu }}$ and $I_{\text {pho }}$, which is estimated as half of the corresponding charged hadronic component.

Photons are selected with $p_{\mathrm{T}}>20 \mathrm{GeV}$ in the pseudorapidity range $|\eta|<1.44$ and $1.57<|\eta|<2.5$. Photon identification is based on the sequential application of several selections. A medium photon identification is used to reconstruct prompt photons (i.e. not from hadron decays) in the final state [20]. The average efficiency for this selection is $80 \%$. Photons selected in this analysis are required to have a narrow transverse shape of the electromagnetic shower, a minimal energy deposit in the HCAL, and to be isolated with respect to other particles. The same isolation variable previously described for the electron selection is used.

The reconstruction, identification, and isolation efficiencies of leptons and photons and the trigger efficiencies of leptons are measured with the "tag-and-probe" technique [23], as a function of particle $\eta$ and $p_{\mathrm{T}}$ in both data and simulation. A sample of events containing a Z boson decaying into $\mathrm{e}^{+} \mathrm{e}^{-}$or $\mu^{+} \mu^{-}$is used for these measurements. The photon efficiencies are derived using a sample of electrons from $\mathrm{Z}$ decays with no requirement on the track and charge of the candidate. These efficiencies are used to correct for the differences between data and simulation.

An event is categorised as a $\mathrm{W}$ decaying to leptons if exactly one electron (muon) with $p_{\mathrm{T}}>35(30) \mathrm{GeV}$ is selected. The selected lepton must match the one that triggered the event and must be associated with the primary vertex of the collision. If the event contains any additional different-flavour leptons or opposite-sign same-flavour leptons, it is excluded from the $\mathrm{W}$ boson candidate sample, but is further checked for the presence of a $\mathrm{Z}$ boson candidate.

An event is categorised as a $\mathrm{Z}$ boson candidate decaying to leptons if two opposite-sign leptons of the same flavour are selected. The leading $p_{\mathrm{T}}$ electron (muon) is required to have $p_{\mathrm{T}}>35(30) \mathrm{GeV}$, and the subleading electron or muon is required to have $p_{\mathrm{T}}>15 \mathrm{GeV}$. Only the leading lepton is required to match the one that triggered the event, although both are required to be associated with the primary vertex of the collision. The invariant mass of the dilepton system is required to be $m_{\ell \ell}>55 \mathrm{GeV}$.

Events are selected if they have a single $\mathrm{W}$ or $\mathrm{Z}$ boson candidate and at least two photons. All reconstructed photons must be separated from each other and from each reconstructed lepton by $\Delta R>0.4$. Photons are discarded if $\left|m_{\mathrm{e}, \gamma}-m_{\mathrm{Z}}\right|<5 \mathrm{GeV}$ (where $m_{\mathrm{e}, \gamma}$ is the invariant mass of an electron and the leading or subleading photon and $m_{\mathrm{Z}}$ 
is the $\mathrm{Z}$ boson invariant mass) or if $\left|m_{\mathrm{e} \gamma \gamma}-m_{\mathrm{Z}}\right|<5 \mathrm{GeV}$ (where $m_{\mathrm{e} \gamma \gamma}$ is the invariant mass of an electron and the two photons). In this way, photons likely coming from finalstate bremsstrahlung radiation are removed and, therefore, the contribution from electrons misidentified as photons is reduced as well.

\section{Background estimation}

The backgrounds in both the $\mathrm{W} \gamma \gamma$ and $\mathrm{Z} \gamma \gamma$ signal regions are categorised as events with a genuine photon or with another object misidentified as a photon. The largest contribution in both channels comes from the misidentification of jets as photons. Another important source of background originates from electrons that are reconstructed as photons because the deposit in the calorimeter is not associated with a track in the tracker. This contribution is particularly relevant in the $\mathrm{W} \gamma \gamma$ electron channel, and is dominated by the $\mathrm{Z} \gamma$ electron channel. Both of these background processes are estimated by exploiting a control sample in data. The remaining minor contributions from processes that have at least one genuine photon $(\mathrm{t} \gamma, \mathrm{t} \overline{\mathrm{t}} \gamma, \mathrm{t} \overline{\mathrm{t}} \gamma \gamma, \mathrm{V} \gamma$ and $\mathrm{VV} \gamma$ ) are estimated using MC simulations and referred to as "others".

The background from events containing nonprompt photons is estimated following a method similar to the ones described in refs. [3, 4]. A W or a $\mathrm{Z}$ boson is selected together with one photon that passes the standard selection except for the isolation requirement both in data and simulation. Events are categorised as "tight" or "loose" if the photon candidate passes or fails the isolation requirement. The probabilities for photon $\epsilon$ and for a jet $f$ to be isolated are computed as

$$
\epsilon=\frac{N_{\gamma, \mathrm{MC}}^{\mathrm{T}}}{N_{\gamma, \mathrm{MC}}^{\mathrm{T}}+N_{\gamma, \mathrm{MC}}^{\mathrm{L}}} \text { and } f=\frac{N_{\gamma, \text { data }}^{\mathrm{T}}}{\mathrm{N}_{\gamma, \text { data }}^{\mathrm{T}}+N_{\gamma, \text { data }}^{\mathrm{L}}},
$$

where $N_{\gamma, \mathrm{MC}}^{\mathrm{T}}\left(N_{\gamma, \mathrm{MC}}^{\mathrm{L}}\right)$ is the number of simulated events with a tight (loose) photon while $N_{\gamma, \text { data }}^{\mathrm{T}}\left(N_{\gamma, \text { data }}^{\mathrm{L}}\right)$ is the number of events with a tight (loose) photon candidate in data after the subtraction of the prompt photon contribution from simulation. These probabilities are calculated separately for photons in the ECAL barrel and endcap regions as a function of the photon $p_{\mathrm{T}}$. The jet-photon misidentification background in the diphoton phase space is then estimated by solving the system

$$
\left(\begin{array}{c}
N_{\mathrm{TT}} \\
N_{\mathrm{TL}} \\
N_{\mathrm{LT}} \\
N_{\mathrm{LL}}
\end{array}\right)=\left(\begin{array}{cccc}
\epsilon_{1} \epsilon_{2} & \epsilon_{1} f_{2} & f_{1} \epsilon_{2} & f_{1} f_{2} \\
\epsilon_{1}\left(1-\epsilon_{2}\right) & \epsilon_{1}\left(1-f_{2}\right) & f_{1}\left(1-\epsilon_{2}\right) & f_{1}\left(1-f_{2}\right) \\
\left(1-\epsilon_{1}\right) \epsilon_{2} & \left(1-\epsilon_{1}\right) f_{2} & \left(1-f_{1}\right) \epsilon_{2} & \left(1-f_{1}\right) f_{2} \\
\left(1-\epsilon_{1}\right)\left(1-\epsilon_{2}\right) & \left(1-\epsilon_{1}\right)\left(1-f_{2}\right) & \left(1-f_{1}\right)\left(1-\epsilon_{2}\right) & \left(1-f_{1}\right)\left(1-f_{2}\right)
\end{array}\right)\left(\begin{array}{c}
\alpha_{\gamma \gamma} \\
\alpha_{\gamma \mathrm{j}} \\
\alpha_{\mathrm{j} \gamma} \\
\alpha_{\mathrm{jj}}
\end{array}\right)
$$

where the indices of the $\epsilon$ and $f$ coefficients refer to the leading and the subleading photon. The $N_{\mathrm{XY}}$ vector contains the number of events where two (TT), one (TL and LT) or zero (LL) photon candidates pass the isolation requirement. The $\alpha_{\mathrm{AB}}$ vector contains the number of signal $(\gamma \gamma)$ and background $(\gamma \mathrm{j}, \mathrm{j} \gamma$ and $\mathrm{jj})$ events. This method is validated in a control region enriched in the jet-photon misidentification background where both photons 
fail the isolation selection. By solving the matrix for each combination of the single photons $p_{\mathrm{T}}$ and $\eta$ and by applying it to the number of events in the double-photon phase space in each diphoton $p_{\mathrm{T}}$ bin one determines the number of background events in the signal region as

$$
N_{\mathrm{TT}}^{\mathrm{j} \rightarrow \gamma}=\sum_{\left(p_{\mathrm{T}}, \eta\right)_{\gamma 1}\left(p_{\mathrm{T}}, \eta\right)_{\gamma 2}}\left(\epsilon_{1} f_{2} \alpha_{\gamma \mathrm{j}}+f_{1} \epsilon_{2} \alpha_{\mathrm{j} \gamma}+f_{1} f_{2} \alpha_{\mathrm{jj}}\right) .
$$

Because of the large contamination from $\mathrm{Z}_{\gamma} \rightarrow$ ee $\gamma$ events where an electron from the $\mathrm{Z}$ boson decay is misclassified as a photon, the jet misidentified as a photon contribution for the $\mathrm{W} \gamma \gamma$ electron channel is estimated from the $\epsilon$ and $f$ coefficients that are evaluated from the single-photon $\mathrm{W} \gamma$ muon sample. For the same reason, the $\mathrm{Z}_{\gamma} \rightarrow$ ee $\gamma$ background contribution has been subtracted from data in the $\mathrm{W} \gamma \gamma$ electron channel before computing the number of background events in the signal region.

To estimate the contribution where an electron is misclassified as a photon, a correction factor is computed from $\mathrm{Z} \gamma$ events and is then applied to the simulation. The invariant mass of an electron and a photon is reconstructed in data and $\mathrm{MC}$ simulation while removing the requirement $\left|m_{\mathrm{e}, \text { lead } \gamma}-m_{\mathrm{Z}}\right|<5 \mathrm{GeV}$. This mass distribution is fitted with the sum of a signal template, derived from MC simulation, and a background function, which has an exponential decay distribution at high mass (above the $\mathrm{Z}$ peak) and a turn-on (linked to the electron and photon $p_{\mathrm{T}}$ thresholds) described by an error function at low mass. A correction factor, obtained in intervals of the photon $p_{\mathrm{T}}$ and $\eta$, is then computed as

$$
\mathcal{F}\left(p_{\mathrm{T}}, \eta\right)=\frac{N_{\text {inv }}^{\text {data }} / N_{\mathrm{Z}}^{\text {data }}}{N_{\text {inv }}^{\mathrm{MC}} / N_{\mathrm{Z}}^{\mathrm{MC}}},
$$

where $N_{\text {inv }}^{\text {data }}$ is the number of events in the electron-photon invariant mass peak obtained by integration of the fitted signal shape and $N_{\mathrm{Z}}^{\text {data }}$ is the number of events for the $\mathrm{Z} \rightarrow$ ee invariant mass distribution obtained by integration of a fitted double-sided Crystal-Ball function [24] in the data. The same procedure is used to calculate the number of events in $\mathrm{MC}$ simulation. By fitting all the distributions in the different $\left(p_{\mathrm{T}}, \eta\right)$ bins, a set of correction factors is computed. All the MC simulations are then corrected for these factors on an event-by-event basis whenever a reconstructed photon matches a generator-level electron. The event-by-event correction factors are on average about $20 \%$.

The pre-fit (i.e. before the fitting procedure described in section 7 ) diphoton $p_{\mathrm{T}}$ distributions for the $\mathrm{W} \gamma \gamma$ and $\mathrm{Z} \gamma \gamma$ analyses, separated in the electron and muon channels, are shown in figure 2. The same distributions obtained in the control region enriched in jets misidentified as photons are shown in figure 3 for the $\mathrm{W} \gamma \gamma$ and $\mathrm{Z} \gamma \gamma$ electron channels. The data and prediction agree thus validating the jet-photon misidentification background estimation procedure. A similar level of agreement is observed in the muon channel.

\section{Systematic uncertainties}

Systematic effects can affect the rates and distributions of both data and simulation. To estimate these uncertainties, the full analysis procedure is repeated by varying each quantity 

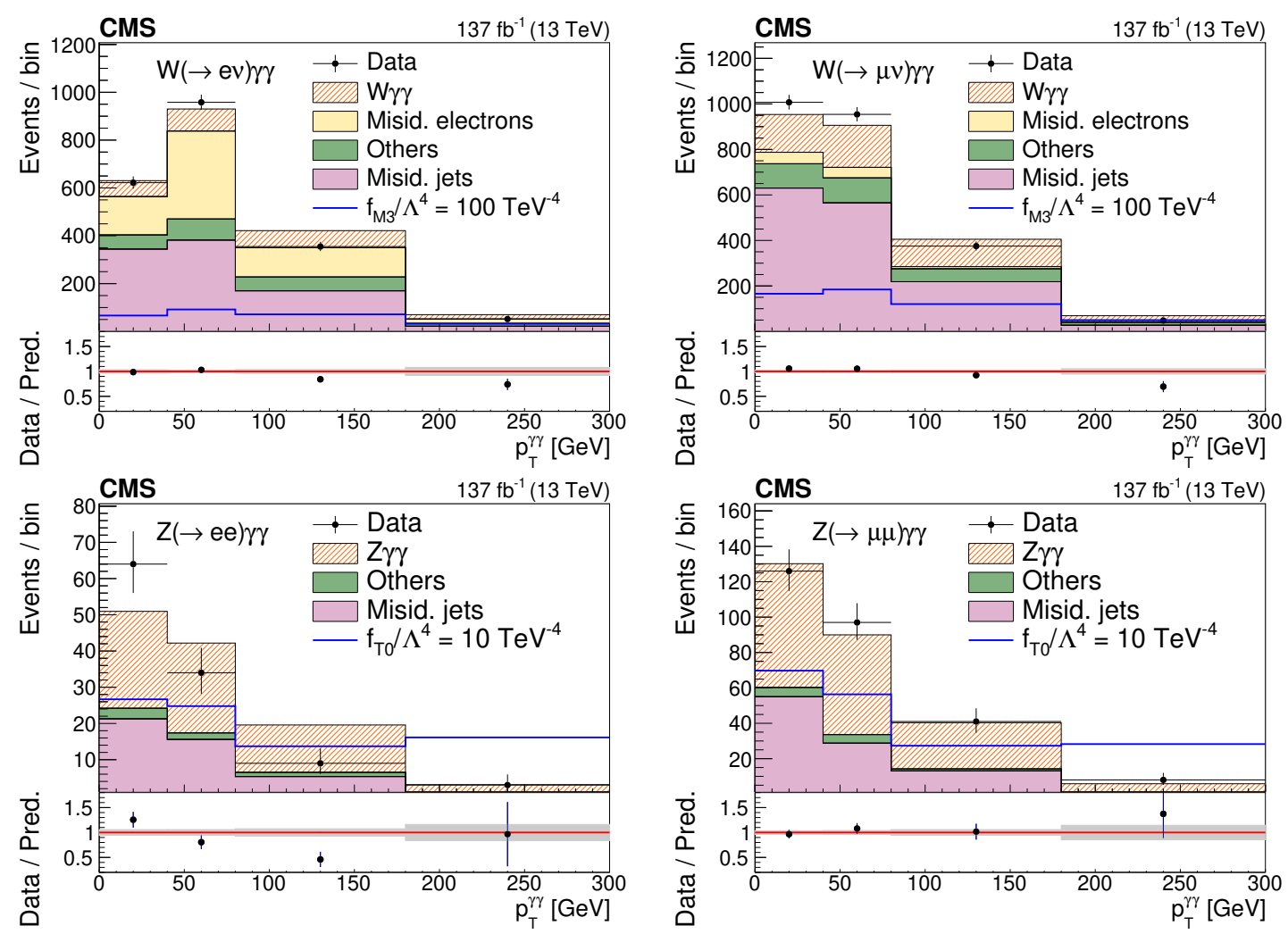

Figure 2. Distribution of the transverse momentum of the diphoton system for the W $\gamma \gamma$ electron (upper left) and muon (upper right) channels and for the $\mathrm{Z} \gamma \gamma$ electron (lower left) and muon (lower right) channels. The predicted yields are shown with their pre-fit normalisations. The black points represent the data with error bars showing the statistical uncertainties. The hatched histogram shows the expected signal contribution. The background estimate for electron (jet) misidentified as photons, obtained from control samples in data, is shown in yellow (purple). The remaining background, derived from MC simulation, is shown in green. In the ratio plots, the grey hashed area is the statistical uncertainty on the sum of signal and backgrounds, while the uncertainty in the black dots is the statistical uncertainty of the data. In blue, the expected distribution for an example value of the anomalous coupling parameters $f_{\mathrm{M} 3} / \Lambda^{4}$ and $f_{\mathrm{T} 0} / \Lambda^{4}$ is also shown (see section 8 for the details).

by plus or minus its standard deviation uncertainty. In this procedure, correlations between the systematic uncertainties are included where appropriate.

The dominant systematic uncertainties come from the estimation of the backgrounds. To determine the systematic uncertainty coming from the jet-photon misidentification background, the same strategy is applied to a QCD control sample that is obtained using the $\mathrm{W} \gamma$ selection but inverting the isolation requirement on the leptons while keeping the photon selection identical to the one for the signal region. This sample is used to obtain an alternative estimate of the jet-photon misidentification background contribution in the $\mathrm{W}$ channel. For the $\mathrm{Z}$ channel, the QCD control sample resulting from the $\mathrm{Z} \gamma$ selection with the inversion of the lepton isolation has insufficient events. Hence, a transfer factor from the $\mathrm{W} \gamma$ selection is computed and applied for the determination of the alternative 

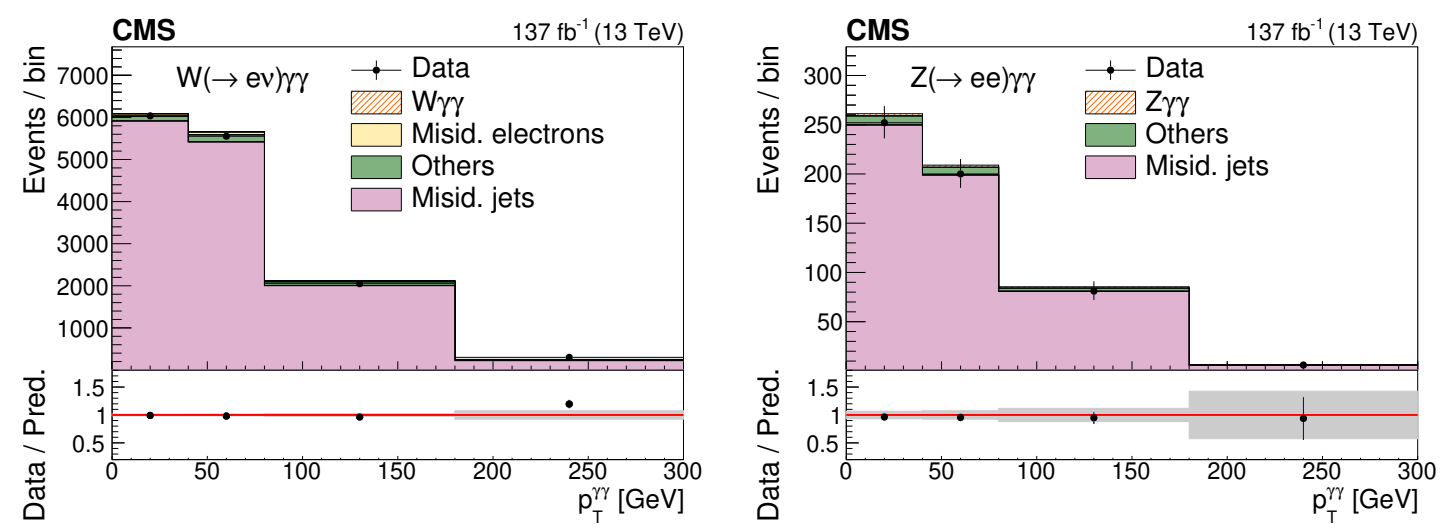

Figure 3. Distribution of the transverse momentum of the diphoton system, obtained in the control region enriched in jets misidentified as photons, for the $\mathrm{W} \gamma \gamma$ and for the $\mathrm{Z} \gamma \gamma$ electron channels. The black points represent the data with error bars showing the statistical uncertainties. The hatched histogram shows the expected negligible signal contribution. The background estimate for electron (jet) misidentified as photons, obtained from control samples in data, is shown in yellow (purple). The remaining backgrounds, derived from MC simulation, are shown in green. In the ratio plots, the grey hashed area is the statistical uncertainty on the sum of signal and backgrounds, while the uncertainty in the black dots is the statistical uncertainty of the data.

estimate of the jet-photon misidentification background contribution in the $\mathrm{Z}$ channel. The systematic uncertainty is computed as half the difference in the distributions between the standard method or the one just described.

Another source of uncertainty in the jet-photon background is related to the modelling of the initial- and final-state radiation and of the energy spectra of the final state particles. An alternative $\mathrm{V} \gamma \mathrm{MC}$ simulation, obtained with SHERPA, is used to evaluate this uncertainty.

The uncertainty in the correction factor related to the background of electrons misidentified as photons is determined by propagating the estimated uncertainty in the correction factor $\mathcal{F}$ of eq. (5.1). This has two components: a statistical one, that comes from the uncertainty in the fitting procedure; and a systematic one that is computed by taking half the difference between the $\mathcal{F}$ factors obtained by performing the fit of the signal component with a double-sided Crystal-Ball function and with the nominal method using an MC template.

The uncertainties in the lepton and photon reconstruction and selection efficiencies are included by computing the cross section with these efficiencies varied up and down by one standard deviation. The uncertainty related to these data-to-simulation corrections is estimated by including the uncertainty in the tag-and-probe method. Uncertainties in the trigger efficiencies are negligible.

The uncertainty in the value of the theoretically computed cross section is accounted for during the subtraction from data of the background processes estimated from MC. Furthermore, the value of the expected cross section has an impact on the estimation of the jet-photon background because the contribution from prompt photons is subtracted from the distribution in data using the $\mathrm{W} \gamma$ and $\mathrm{Z} \gamma$ simulations. To estimate these contribu- 


\begin{tabular}{ccccccc}
\hline Systematic source & $\mathrm{e} v_{\mathrm{e}} \gamma \gamma[\%]$ & $\mu \nu_{\mu} \gamma \gamma[\%]$ & $\ell \nu \gamma \gamma[\%]$ & $\mathrm{ee} \gamma \gamma[\%]$ & $\mu \mu \gamma \gamma[\%]$ & $\ell \ell \gamma[\%]$ \\
\hline Integrated luminosity & $<1$ & 2 & 2 & 3 & 1 & 3 \\
Pile-up & 2 & $<1$ & $<1$ & 2 & $<1$ & 1 \\
Electron efficiencies & 4 & - & $<1$ & 3 & - & 1 \\
Muon efficiencies & 1 & $<1$ & $<1$ & 2 & $<1$ & 1 \\
Photon efficiencies & 18 & 13 & 12 & 6 & 5 & 5 \\
Jet-photon misid. & 25 & 22 & 21 & 6 & 5 & 6 \\
Electron-photon misid. & 4 & $<1$ & $<1$ & - & - & - \\
$\mathrm{W} \gamma$ theoretical cross section & 3 & 3 & 3 & $<1$ & $<1$ & $<1$ \\
$\mathrm{Z} \gamma$ theoretical cross section & 4 & $<1$ & $<1$ & 7 & 5 & 6 \\
Other bkgs theoretical cross section & 5 & 2 & 2 & $<1$ & $<1$ & $<1$ \\
Simulated sample event count & 18 & 7 & 8 & 7 & 3 & 4 \\
\hline
\end{tabular}

Table 1. Summary of the systematic uncertainties (in percent) for the $\mathrm{W} \gamma \gamma$ and $\mathrm{Z} \gamma \gamma$ cross section measurements. The numbers indicate the impact of each systematic uncertainty in the value of the measured cross section in the corresponding channel. The systematic uncertainties in the jets misidentified as photons are added in quadrature in the table.

tions, the cross sections of the $\mathrm{W} \gamma, \mathrm{Z} \gamma$, and of the other minor backgrounds are varied independently. The uncertainty in the $\mathrm{Z} \gamma$ cross section is estimated as half the difference between the next-to-NLO and the NLO values computed with MATRIX [25], and amounts to $2.5 \%$. The same uncertainty is assumed for the $\mathrm{W} \gamma$ cross section, and a value of $7.5 \%$ is used for the other simulated backgrounds.

The total inelastic cross section is varied by $4.6 \%$ [26] to estimate the impact on the final result of the pileup reweighting procedure. The uncertainty because of the integrated luminosity measurement is equal to 2.5, 2.3, and 2.5\% for the 2016, 2017 and 2018 data taking periods, respectively [27-30]. Because of the uncorrelated time evolution of some systematic uncertainties, the total integrated luminosity has an uncertainty of $1.8 \%$ and is applied to all the processes estimated with an MC simulation. The effect of the uncertainty in the integrated luminosity affects the estimation of the jet-photon misidentification background as well as the MC contributions in the diphoton distributions.

For the extraction of the results, each systematic uncertainty is represented by a nuisance parameter, which affects the shape and the normalisation of the distribution of the various background contributions. The variation of the nuisance parameter results in a continuous perturbation of the spectrum, following a Gaussian probability density function. The impact of each systematic uncertainty is obtained by freezing the set of associated nuisance parameters to their best-fit values and comparing the total uncertainty in the measured cross section with the result from the nominal fit [31]. The contributions of the different systematic uncertainties for both the $\mathrm{W} \gamma \gamma$ and $\mathrm{Z} \gamma \gamma$ processes are presented in table 1.

\section{Cross section measurements}

The cross sections for the $\mathrm{W} \gamma \gamma$ and $\mathrm{Z} \gamma \gamma$ processes are measured separately in the electron and muon channels using a sample of events corresponding to an integrated luminosity of 


\begin{tabular}{ccc}
\hline Process & $\mathrm{e} v_{\mathrm{e} \gamma} \gamma$ & $\mu v_{\mu} \gamma \gamma$ \\
\hline Misid. jets & $918 \pm 23$ (stat) \pm 180 (syst) & $1441 \pm 27$ (stat) \pm 280 (syst) \\
Misid. electrons & $669 \pm 28$ (stat) \pm 34 (syst) & $107 \pm 9$ (stat) \pm 7 (syst) \\
Others & $217 \pm 11$ (stat) \pm 20 (syst) & $286 \pm 11$ (stat) \pm 25 (syst) \\
Total backgrounds & $1804 \pm 38$ (stat) \pm 180 (syst) & $1834 \pm 30$ (stat) \pm 280 (syst) \\
Expected signal & $248 \pm 6$ (stat) \pm 17 (syst) & $500 \pm 8$ (stat) \pm 33 (syst) \\
Total prediction & $2052 \pm 38$ (stat) \pm 180 (syst) & $2334 \pm 31$ (stat) \pm 280 (syst) \\
Data & 1987 & 2384 \\
\hline Process & ee $\gamma \gamma$ & $\mu \mu \gamma \gamma$ \\
\hline Misid. jets & $42 \pm 4$ (stat) \pm 9 (syst) & $98 \pm 5$ (stat) \pm 27 (syst) \\
Others & $6 \pm 1$ (stat) \pm 1 (syst) & $11 \pm 2$ (stat) \pm 1 (syst) \\
Total backgrounds & $48 \pm 4$ (stat) \pm 9 (syst) & $109 \pm 6$ (stat) \pm 27 (syst) \\
Expected signal & $68 \pm 2$ (stat) \pm 5 (syst) & $157 \pm 3$ (stat) \pm 11 (syst) \\
Total prediction & $116 \pm 4$ (stat) \pm 8 (syst) & $266 \pm 6$ (stat) \pm 23 (syst) \\
Data & 110 & 272 \\
\hline
\end{tabular}

Table 2. Summary of the pre-fit predicted and observed numbers of events for $137 \mathrm{fb}^{-1}$ for the $\mathrm{W} \gamma \gamma$ (upper table) and $\mathrm{Z} \gamma \gamma_{\gamma}$ (lower table) selections in the electron and muon channels. The systematic uncertainties of the individual backgrounds and the total background are obtained by summing the contributions of different systematic uncertainties in quadrature. The statistical uncertainties are those related to the MC event samples and control region statistical uncertainties.

$137 \mathrm{fb}^{-1}$ (LHC Run 2 data). The observed and predicted numbers of events are presented in table 2 .

The measured yields in the electron and muon channels are extrapolated to a common fiducial phase space determined from simulated signal events at the generated particle level. Generated particles are considered stable if their mean decay length is larger than $1 \mathrm{~cm}$. Generated leptons are required to have a $p_{\mathrm{T}}>15 \mathrm{GeV}$ and $|\eta|<2.5$. The momenta of photons in a cone of $\Delta R=0.1$, the same cone size as the one applied to reconstructed data, are added to the charged lepton momentum to correct for final-state radiation. Generated photons are required to have $p_{\mathrm{T}}>15 \mathrm{GeV}$ and $|\eta|<2.5$. Additionally, the candidate photons are required to have no selected leptons or photons in a cone of radius $\Delta R=0.4$ and no other stable particles, apart from photons and neutrinos, in a cone of radius $\Delta R=0.1$. Events are then selected in the $\mathrm{W} \gamma \gamma$ channel by requiring exactly one electron (muon) with $p_{\mathrm{T}}>30 \mathrm{GeV}$ and at least two photons with $p_{\mathrm{T}}>20 \mathrm{GeV}$. Events are selected in the $\mathrm{Z} \gamma \gamma$ channel by requiring two electrons (muons), at least one of them with $p_{\mathrm{T}}>30 \mathrm{GeV}$, and not less than two photons, each of them with $p_{\mathrm{T}}>20 \mathrm{GeV}$. Additionally, the invariant mass of the dilepton system is required to be $m_{\ell \ell}>55 \mathrm{GeV}$.

The expected theoretical cross sections are predicted at NLO and their uncertainties come from the finite MC sample event count used to compute them, the PDF set, the 
factorisation and renormalisation scales. Statistical uncertainties are estimated to be of the order of $0.2 \%$ in both the $\mathrm{W} \gamma \gamma$ and $\mathrm{Z} \gamma \gamma$ channels. Uncertainties related to the PDF set are estimated using a set of 100 replicas of the NNPDF 3.1 PDF set, following the ref. [12] prescription, and are estimated to be of the order of $0.3 \%$ in the e $v \gamma \gamma$ and $\mu \nu \gamma \gamma$ and of $0.8 \%$ in the ee $\gamma \gamma$ and $\mu \mu \gamma \gamma$ channels. Uncertainties related to the renormalisation and factorisation scale choice are estimated by independently varying $\mu_{\mathrm{R}}$ and $\mu_{\mathrm{F}}$ by a factor of 0.5 and 2 , with the condition that $1 / 2<\mu_{\mathrm{R}} / \mu_{\mathrm{F}}<2$. The uncertainties are defined as the maximal differences from the nominal values and are estimated to be of the order of $0.6(0.5) \%$ in the e $v \gamma \gamma(\mu \nu \gamma \gamma)$ and of $0.6(0.7) \%$ in the ee $\gamma \gamma(\mu \mu \gamma \gamma)$ channels. Uncertainties related to the value of the strong coupling are estimated to be of the order of $0.03(0.02) \%$ in the e $v \gamma \gamma(\mu \nu \gamma \gamma)$ and of $0.4 \%$ in the ee $\gamma \gamma$ and $\mu \mu \gamma \gamma$ channels.

Binned maximum likelihood fits to the diphoton $p_{\mathrm{T}}$ distributions in figure 2 are performed to extract the signal strength $\mu$ and the significance of the results [32, 33]. The results are obtained separately in the electron, muon and lepton channels. The systematic uncertainties and the statistical uncertainty in the MC predictions are treated as nuisance parameters in the fits and profiled. The high $p_{\mathrm{T}}$ bins in the distributions are the more relevant ones for the determination of the limits.

The measured cross sections are obtained by multiplying the observed signal strength $\mu$ by the expected theoretical cross section of the signal MC simulated sample. The theoretical cross section for the $\mathrm{W} \gamma \gamma$ and $\mathrm{Z} \gamma \gamma$ signals obtained from MADGRAPH5_amC@NLO at NLO accuracy are $18.70 \pm 0.03$ (MC stat) \pm 0.12 (PDF + scale) fb and 5.96 \pm 0.01 (MC stat) \pm $0.06(\mathrm{PDF}+$ scale $) \mathrm{fb}$, respectively.

In the electron channel, the best fit value for the $\mathrm{W} \gamma \gamma$ signal strength is $0.23_{-0.22}^{+0.22}$ (stat) ${ }_{-0.30}^{+0.32}$ (syst) and for the $\mathrm{Z} \gamma \gamma$ signal strength is $0.73_{-0.17}^{+0.18}$ (stat) ${ }_{-0.13}^{+0.12}$ (syst). The measured cross sections are:

$$
\begin{aligned}
\sigma(\mathrm{W} \gamma \gamma)_{\mathrm{e} \nu}^{\text {meas }} & =4.4_{-4.1}^{+4.1}(\text { stat })_{-5.5}^{+6.0}(\text { syst }) \pm 0.03(\mathrm{PDF}+\mathrm{scale}) \mathrm{fb}, \\
\sigma(\mathrm{Z} \gamma \gamma)_{\mathrm{ee}}^{\text {meas }} & =4.35_{-0.99}^{+1.05}(\text { stat })_{-0.77}^{+0.71} \text { (syst) } \pm 0.05(\mathrm{PDF}+\text { scale }) \mathrm{fb} .
\end{aligned}
$$

In the muon channel, the best fit value for the $\mathrm{W} \gamma \gamma$ signal strength is $0.74_{-0.11}^{+0.11}$ (stat $)_{-0.22}^{+0.23}$ (syst) and for the $\mathrm{Z} \gamma \gamma$ signal strength is $1.06_{-0.11}^{+0.11}$ (stat) ${ }_{-0.10}^{+0.10}$ (syst). The measured cross sections are:

$$
\begin{aligned}
\sigma(\mathrm{W} \gamma \gamma)_{\mu \nu}^{\text {meas }} & =13.8_{-2.1}^{+2.1}(\text { stat })_{-4.2}^{+4.3}(\text { syst }) \pm 0.08(\mathrm{PDF}+\text { scale }) \mathrm{fb}, \\
\sigma(\mathrm{Z} \gamma \gamma)_{\mu \mu}^{\text {meas }} & =6.29_{-0.64}^{+0.67}(\text { stat })_{-0.58}^{+0.57}(\text { syst }) \pm 0.07(\mathrm{PDF}+\text { scale }) \mathrm{fb} .
\end{aligned}
$$

The results of the fit for the electron and muon channels separately are compatible within two sigmas. In the combined electron and muon channel, the best fit value for the $\mathrm{W} \gamma \gamma$ signal strength is $0.73_{-0.10}^{+0.10}$ (stat) ${ }_{-0.22}^{+0.22}$ (syst) and for the $\mathrm{Z} \gamma \gamma$ signal strength is $0.91_{-0.09}^{+0.10}$ (stat) ${ }_{-0.12}^{+0.11}$ (syst). The measured cross sections are:

$$
\begin{aligned}
\sigma(\mathrm{W} \gamma \gamma)^{\text {meas }} & =13.6_{-1.9}^{+1.9}(\text { stat })_{-4.0}^{+4.0}(\text { syst }) \pm 0.08(\mathrm{PDF}+\text { scale }) \mathrm{fb}, \\
\sigma(\mathrm{Z} \gamma \gamma)^{\text {meas }} & =5.41_{-0.55}^{+0.58}(\text { stat })_{-0.70}^{+0.64}(\text { syst }) \pm 0.06(\mathrm{PDF}+\text { scale }) \mathrm{fb} .
\end{aligned}
$$

The sensitivity for the $\mathrm{W} \gamma \gamma$ cross section measurement is dominated by the muon channel. The measured signal strengths are summarised in figure 4. 

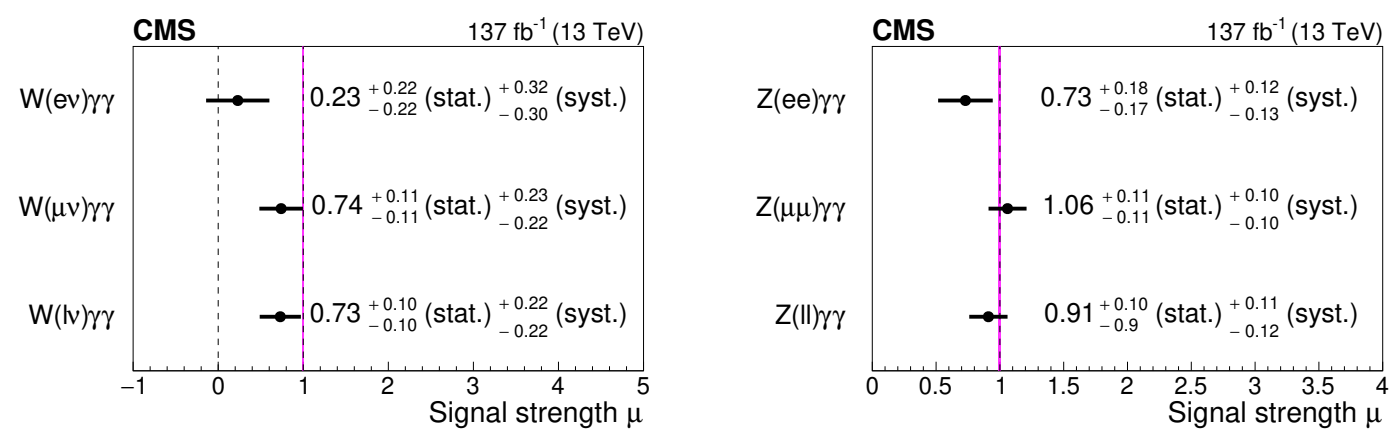

Figure 4. Best fit values of the signal strengths for the $\mathrm{W} \gamma \gamma$ (left) and $\mathrm{Z} \gamma \gamma$ (right) channels. The error bars represent the total uncertainty while the magenta bands represent the theoretical uncertainty in the MADGRAPH5_aMC@NLO cross section.

The significance of the cross section measurement for both the $\mathrm{W} \gamma \gamma$ and $\mathrm{Z} \gamma \gamma$ channels is quantified using the background-only hypothesis under the asymptotic approximation [34]. The observed (expected) significance for the $\mathrm{W} \gamma \gamma$ signal is $0.6(2.7) \sigma$ in the electron channel and 3.0 (4.3) $\sigma$ in the muon channel; for the $\mathrm{Z} \gamma \gamma$ is 3.4 (5.0) $\sigma$ in the electron channel and 5.4(5.1) $\sigma$ in the muon channel; the combined significance for the $\mathrm{W} \gamma \gamma$ is 3.1 (4.5) $\sigma$ and for the $\mathrm{Z} \gamma \gamma$ is $4.8(5.8) \sigma$.

\section{Limits on anomalous quartic gauge couplings}

Studies of the anomalous gauge couplings can be performed in the EFT framework [1] by expanding the SM Lagrangian to include terms with dimension higher than four. In particular, both the $\mathrm{W} \gamma \gamma$ and $\mathrm{Z} \gamma \gamma$ processes are sensitive to the presence of dimension- 6 and dimension-8 operators [35]. Because of the available statistics in the $\mathrm{V} \gamma \gamma$ channel, the sensitivity to dimension- 6 operators is expected to be lower than the one in the diboson production. The contribution of each operator is proportional to a coupling constant $f$ and to the inverse of the energy scale $\Lambda$ at which the new phenomena appear.

In the generation of the anomalous couplings samples, a calculation using 10 (8) different dimension- 8 operators was performed for the $\mathrm{W} \gamma \gamma(\mathrm{Z} \gamma \gamma)$ process. The operators can be divided into two subsets: the $\mathcal{L}_{\mathrm{M} 0}-\mathcal{L}_{\mathrm{M} 7}$ ones, that contain both the $\mathrm{SU}(2)_{L}$ and $\mathrm{U}(1)_{Y}$ field strengths and the covariant derivative of the Higgs doublet, and the $\mathcal{L}_{\mathrm{T} 0}-\mathcal{L}_{\mathrm{T} 9}$ ones, that contain only the two field strengths. In particular, the $\mathrm{W} \gamma \gamma$ channel is especially sensitive to the M2, M3, T0, T1, T2, T5, T6, and T7 operators, whereas the $\mathrm{Z} \gamma \gamma$ channel is especially sensitive to the $\mathrm{T} 0, \mathrm{~T} 1, \mathrm{~T} 2, \mathrm{~T} 5, \mathrm{~T} 6, \mathrm{~T} 7, \mathrm{~T} 8$, and $\mathrm{T} 9$ operators.

The distribution of the $p_{\mathrm{T}}$ of the diphoton system (shown in figure 2) is used to constrain the aQGC parameters under the hypothesis of absence of anomalies in triple gauge couplings. The contribution of aQGCs is enhanced at high values of the $p_{\mathrm{T}}$ of the diphoton system. The distribution of the aQGCs as a function of the couplings themselves has a quadratic behaviour, and hence a parabolic fit is implemented to interpolate between the different values obtained via the parameter scan. The fitting procedure is performed bin-by-bin to exploit the shape of the distribution to set the limits and include the different 


\begin{tabular}{ccccc}
\hline & \multicolumn{2}{c}{$\mathrm{W} \gamma \gamma\left(\mathrm{TeV}^{-4}\right)$} & \multicolumn{2}{c}{$\mathrm{Z} \gamma \gamma\left(\mathrm{TeV}^{-4}\right)$} \\
Parameter & Expected & Observed & Expected & Observed \\
\hline$f_{\mathrm{M} 2} / \Lambda^{4}$ & {$[-57.3,57.1]$} & {$[-39.9,39.5]$} & - & - \\
$f_{\mathrm{M} 3} / \Lambda^{4}$ & {$[-91.8,92.6]$} & {$[-63.8,65.0]$} & - & - \\
$f_{\mathrm{T} 0} / \Lambda^{4}$ & {$[-1.86,1.86]$} & {$[-1.30,1.30]$} & {$[-4.86,4.66]$} & {$[-5.70,5.46]$} \\
$f_{\mathrm{T} 1} / \Lambda^{4}$ & {$[-2.38,2.38]$} & {$[-1.70,1.66]$} & {$[-4.86,4.66]$} & {$[-5.70,5.46]$} \\
$f_{\mathrm{T} 2} / \Lambda^{4}$ & {$[-5.16,5.16]$} & {$[-3.64,3.64]$} & {$[-9.72,9.32]$} & {$[-11.4,10.9]$} \\
$f_{\mathrm{T} 5} / \Lambda^{4}$ & {$[-0.76,0.84]$} & {$[-0.52,0.60]$} & {$[-2.44,2.52]$} & {$[-2.92,2.92]$} \\
$f_{\mathrm{T} 6} / \Lambda^{4}$ & {$[-0.92,1.00]$} & {$[-0.60,0.68]$} & {$[-3.24,3.24]$} & {$[-3.80,3.88]$} \\
$f_{\mathrm{T} 7} / \Lambda^{4}$ & {$[-1.64,1.72]$} & {$[-1.16,1.16]$} & {$[-6.68,6.60]$} & {$[-7.88,7.72]$} \\
$f_{\mathrm{T} 8} / \Lambda^{4}$ & - & - & {$[-0.90,0.94]$} & {$[-1.06,1.10]$} \\
$f_{\mathrm{T} 9} / \Lambda^{4}$ & - & - & {$[-1.54,1.54]$} & {$[-1.82,1.82]$} \\
\hline
\end{tabular}

Table 3. Expected and observed $95 \%$ confidence level intervals for the different anomalous couplings in both the $\mathrm{W} \gamma \gamma$ and $\mathrm{Z} \gamma \gamma$ channels.

systematic uncertainties. To further increase the sensitivity, electron and muon channels are combined. Each operator coefficient is scanned independently with all other operators set to zero. The extraction of the $95 \%$ confidence level upper and lower limits on the aQGCs is performed by exploiting the procedure described in ref. [33]. The expected and measured limits for both the $\mathrm{W} \gamma \gamma$ and $\mathrm{Z} \gamma \gamma$ processes are presented in table 3 .

In particular, the intervals computed for the $f_{\mathrm{T} 5}$ and $f_{\mathrm{T} 6}$ parameters are the most constraining ones in the $\mathrm{W} \gamma \gamma$ channel and are comparable to the most stringent results obtained by the W $\gamma \mathrm{jj}$ [36] analysis of the CMS collaboration at $13 \mathrm{TeV}$. The intervals computed for the $f_{\mathrm{T} 0}$ and $f_{\mathrm{T} 5}$ parameters in the $\mathrm{W} \gamma \gamma$ channel are more stringent than the ones obtained by the $\mathrm{Z}_{\gamma \gamma}$ [3] and $\mathrm{Z} \gamma \mathrm{jj}$ [37] analyses of ATLAS at $8 \mathrm{TeV}$. For the $\mathrm{Z} \gamma \gamma$ channel, the most stringent interval is the one computed for the $f_{\mathrm{T} 9}$ parameter, which is competitive with the results obtained by the Z $\gamma \mathrm{jj}$ [38] and ZZjj [39] analyses of CMS at $13 \mathrm{TeV}$. The intervals computed for the $f_{\mathrm{T} 8}$ and $f_{\mathrm{T} 9}$ parameters in the $\mathrm{Z} \gamma \gamma$ channel are more stringent than the ones obtained by the $\mathrm{Z}_{\gamma \gamma}$ and $\mathrm{Z} \gamma \mathrm{jj}$ analyses of ATLAS at $8 \mathrm{TeV}$.

\section{Summary}

The cross sections for both the $\mathrm{W} \gamma \gamma$ and $\mathrm{Z} \gamma \gamma$ processes are measured in proton-proton collisions by the CMS experiment at a centre-of-mass energy of $13 \mathrm{TeV}$ corresponding to an integrated luminosity of $137 \mathrm{fb}^{-1}$.

The cross sections are measured in a fiducial region where simulated signal events are selected at generator level in the $\mathrm{W} \gamma \gamma$ channel by requiring exactly one electron or muon with transverse momentum $p_{\mathrm{T}}>30 \mathrm{GeV}$ and at least two photons, each with $p_{\mathrm{T}}>20 \mathrm{GeV}$. Events are selected in the $\mathrm{Z} \gamma \gamma$ channel by requiring two oppositely charged electrons or muons, at least one of them with $p_{\mathrm{T}}>30 \mathrm{GeV}$, and at least two photons, each with 
$p_{\mathrm{T}}>20 \mathrm{GeV}$. All leptons and photons are required to have pseudorapidity $|\eta|<2.5$. Additionally, the invariant mass of the dilepton system is required to exceed $m_{\ell \ell}>55 \mathrm{GeV}$.

The measured cross sections are $13.6_{-1.9}^{+1.9}$ (stat) $)_{-4.0}^{+4.0}$ (syst) \pm 0.08 (PDF + scale) fb for the $\mathrm{W} \gamma \gamma$ channel and $5.41_{-0.55}^{+0.58}$ (stat) $)_{-0.70}^{+0.64}$ (syst) \pm 0.06 (PDF + scale) fb for the $\mathrm{Z} \gamma \gamma$ channel. These results are in agreement with the theoretical cross sections computed at next-toleading order. The corresponding signal significances are 3.1 and 4.8 standard deviations. Limits on anomalous quartic gauge couplings are set using both channels.

\section{Acknowledgments}

We congratulate our colleagues in the CERN accelerator departments for the excellent performance of the LHC and thank the technical and administrative staffs at CERN and at other CMS institutes for their contributions to the success of the CMS effort. In addition, we gratefully acknowledge the computing centres and personnel of the Worldwide LHC Computing Grid and other centres for delivering so effectively the computing infrastructure essential to our analyses. Finally, we acknowledge the enduring support for the construction and operation of the LHC, the CMS detector, and the supporting computing infrastructure provided by the following funding agencies: BMBWF and FWF (Austria); FNRS and FWO (Belgium); CNPq, CAPES, FAPERJ, FAPERGS, and FAPESP (Brazil); MES (Bulgaria); CERN; CAS, MoST, and NSFC (China); MINCIENCIAS (Colombia); MSES and CSF (Croatia); RIF (Cyprus); SENESCYT (Ecuador); MoER, ERC PUT and ERDF (Estonia); Academy of Finland, MEC, and HIP (Finland); CEA and CNRS/IN2P3 (France); BMBF, DFG, and HGF (Germany); GSRT (Greece); NKFIA (Hungary); DAE and DST (India); IPM (Iran); SFI (Ireland); INFN (Italy); MSIP and NRF (Republic of Korea); MES (Latvia); LAS (Lithuania); MOE and UM (Malaysia); BUAP, CINVESTAV, CONACYT, LNS, SEP, and UASLP-FAI (Mexico); MOS (Montenegro); MBIE (New Zealand); PAEC (Pakistan); MSHE and NSC (Poland); FCT (Portugal); JINR (Dubna); MON, RosAtom, RAS, RFBR, and NRC KI (Russia); MESTD (Serbia); SEIDI, CPAN, PCTI, and FEDER (Spain); MOSTR (Sri Lanka); Swiss Funding Agencies (Switzerland); MST (Taipei); ThEPCenter, IPST, STAR, and NSTDA (Thailand); TUBITAK and TAEK (Turkey); NASU (Ukraine); STFC (U.K.); DOE and NSF (U.S.A.).

Individuals have received support from the Marie-Curie programme and the European Research Council and Horizon 2020 Grant, contract Nos. 675440, 724704, 752730, 765710 and 824093 (European Union); the Leventis Foundation; the Alfred P. Sloan Foundation; the Alexander von Humboldt Foundation; the Belgian Federal Science Policy Office; the Fonds pour la Formation à la Recherche dans l'Industrie et dans l'Agriculture (FRIA-Belgium); the Agentschap voor Innovatie door Wetenschap en Technologie (IWTBelgium); the F.R.S.-FNRS and FWO (Belgium) under the "Excellence of Science - EOS" - be.h project n. 30820817; the Beijing Municipal Science \& Technology Commission, No. Z191100007219010; the Ministry of Education, Youth and Sports (MEYS) of the Czech Republic; the Deutsche Forschungsgemeinschaft (DFG), under Germany's Excellence Strategy - EXC 2121 "Quantum Universe" — 390833306, and under project number 400140256 — GRK2497; the Lendület ("Momentum") Programme and the János Bolyai Research 
Scholarship of the Hungarian Academy of Sciences, the New National Excellence Program ÚNKP, the NKFIA research grants 123842, 123959, 124845, 124850, 125105, 128713, 128786, and 129058 (Hungary); the Council of Science and Industrial Research, India; the Latvian Council of Science; the Ministry of Science and Higher Education and the National Science Center, contracts Opus 2014/15/B/ST2/03998 and 2015/19/B/ST2/02861 (Poland); the National Priorities Research Program by Qatar National Research Fund; the Ministry of Science and Higher Education, project no. 0723-2020-0041 (Russia); the Programa Estatal de Fomento de la Investigación Científica y Técnica de Excelencia María de Maeztu, grant MDM-2015-0509 and the Programa Severo Ochoa del Principado de Asturias; the Thalis and Aristeia programmes cofinanced by EU-ESF and the Greek NSRF; the Rachadapisek Sompot Fund for Postdoctoral Fellowship, Chulalongkorn University and the Chulalongkorn Academic into Its 2nd Century Project Advancement Project (Thailand); the Kavli Foundation; the Nvidia Corporation; the SuperMicro Corporation; the Welch Foundation, contract C-1845; and the Weston Havens Foundation (U.S.A.).

Open Access. This article is distributed under the terms of the Creative Commons Attribution License (CC-BY 4.0), which permits any use, distribution and reproduction in any medium, provided the original author(s) and source are credited.

\section{References}

[1] C. Degrande et al., Effective Field Theory: A Modern Approach to Anomalous Couplings, Annals Phys. 335 (2013) 21 [arXiv:1205.4231] [INSPIRE].

[2] ATLAS collaboration, Evidence of $W \gamma \gamma$ Production in pp Collisions at $\sqrt{s}=8 \mathrm{TeV}$ and Limits on Anomalous Quartic Gauge Couplings with the ATLAS Detector, Phys. Rev. Lett. 115 (2015) 031802 [arXiv:1503.03243] [INSPIRE].

[3] ATLAS collaboration, Measurements of $Z \gamma$ and $Z \gamma \gamma$ production in pp collisions at $\sqrt{s}=8 \mathrm{TeV}$ with the ATLAS detector, Phys. Rev. D 93 (2016) 112002 [arXiv:1604.05232] [INSPIRE].

[4] CMS collaboration, Measurements of the $p p \rightarrow W \gamma \gamma$ and $p p \rightarrow Z \gamma \gamma$ cross sections and limits on anomalous quartic gauge couplings at $\sqrt{s}=8 \mathrm{TeV}$, JHEP 10 (2017) 072 [arXiv: 1704.00366] [INSPIRE].

[5] G. Bozzi, F. Campanario, M. Rauch and D. Zeppenfeld, $W^{ \pm} \gamma \gamma$ production with leptonic decays at NLO QCD, Phys. Rev. D 83 (2011) 114035 [arXiv:1103.4613] [InSPIRE].

[6] U. Baur, D. Wackeroth and M.M. Weber, Radiative corrections to $W \gamma \gamma$ production at the LHC, PoS RAD COR2009 (2010) 067 [arXiv:1001.2688] [INSPIRE].

[7] G. Bozzi, F. Campanario, M. Rauch and D. Zeppenfeld, $Z \gamma \gamma$ production with leptonic decays and triple photon production at next-to-leading order QCD, Phys. Rev. D 84 (2011) 074028 [arXiv:1107.3149] [INSPIRE].

[8] CMS collaboration, The CMS trigger system, 2017 JINST 12 P01020 [arXiv:1609.02366] [INSPIRE].

[9] CMS collaboration, The CMS Experiment at the CERN LHC, 2008 JINST 3 S08004 [INSPIRE]. 
[10] J. Alwall et al., The automated computation of tree-level and next-to-leading order differential cross sections, and their matching to parton shower simulations, JHEP 07 (2014) 079 [arXiv: 1405.0301] [INSPIRE].

[11] NNPDF collaboration, Parton distributions for the LHC Run II, JHEP 04 (2015) 040 [arXiv: 1410.8849] [INSPIRE].

[12] NNPDF collaboration, Parton distributions from high-precision collider data, Eur. Phys. J. $C 77$ (2017) 663 [arXiv:1706.00428] [InSPIRE].

[13] T. Gleisberg, S. Hoeche, F. Krauss, A. Schalicke, S. Schumann and J.-C. Winter, SHERPA

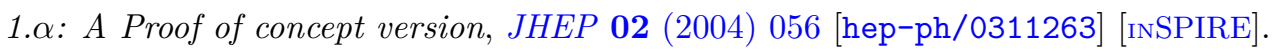

[14] E. Bothmann et al., Event Generation with Sherpa 2.2, SciPost Phys. 7 (2019) 034 [arXiv: 1905.09127] [INSPIRE].

[15] CMS collaboration, Event generator tunes obtained from underlying event and multiparton scattering measurements, Eur. Phys. J. C 76 (2016) 155 [arXiv:1512.00815] [INSPIRE].

[16] CMS collaboration, Extraction and validation of a new set of CMS PYTHIA8 tunes from underlying-event measurements, Eur. Phys. J. C 80 (2020) 4 [arXiv:1903.12179] [INSPIRE].

[17] C. Degrande, C. Duhr, B. Fuks, D. Grellscheid, O. Mattelaer and T. Reiter, UFO - The Universal FeynRules Output, Comput. Phys. Commun. 183 (2012) 1201 [arXiv:1108.2040] [INSPIRE].

[18] GEANT4 collaboration, GEANT4 - a simulation toolkit, Nucl. Instrum. Meth. A 506 (2003) 250 [INSPIRE].

[19] CMS collaboration, Particle-flow reconstruction and global event description with the CMS detector, 2017 JINST 12 P10003 [arXiv: 1706. 04965] [INSPIRE].

[20] CMS collaboration, Electron and photon reconstruction and identification with the CMS experiment at the CERN LHC, 2021 JINST 16 P05014 [arXiv:2012.06888] [INSPIRE].

[21] M. Cacciari and G.P. Salam, Pileup subtraction using jet areas, Phys. Lett. B 659 (2008) 119 [arXiv: 0707.1378] [INSPIRE].

[22] CMS collaboration, Performance of the CMS muon detector and muon reconstruction with proton-proton collisions at $\sqrt{s}=13 \mathrm{TeV}, 2018$ JINST $13 \mathrm{P} 06015$ [arXiv:1804.04528] [INSPIRE].

[23] CMS collaboration, Measurement of the Inclusive $W$ and $Z$ Production Cross Sections in pp Collisions at $\sqrt{s}=7 \mathrm{TeV}$, JHEP 10 (2011) 132 [arXiv:1107.4789] [INSPIRE].

[24] M.J. Oreglia, A study of the reactions $\psi^{\prime} \rightarrow \gamma \gamma \psi$, Ph.D. Thesis, Stanford University, Stanford CA U.S.A. (1980).

[25] M. Grazzini, S. Kallweit and M. Wiesemann, Fully differential NNLO computations with MATRIX, Eur. Phys. J. C 78 (2018) 537 [arXiv:1711.06631] [InSPIRE].

[26] CMS collaboration, Measurement of the inelastic proton-proton cross section at $\sqrt{s}=13 \mathrm{TeV}$, JHEP 07 (2018) 161 [arXiv: 1802.02613] [INSPIRE].

[27] CMS collaboration, CMS Luminosity Measurements for the 2016 Data Taking Period, CMS-PAS-LUM-17-001 (2017).

[28] CMS collaboration, CMS luminosity measurement for the 2017 data-taking period at $\sqrt{s}=13 \mathrm{TeV}$, CMS-PAS-LUM-17-004 (2018). 
[29] CMS collaboration, CMS luminosity measurement for the 2018 data-taking period at $\sqrt{s}=13 \mathrm{TeV}$, CMS-PAS-LUM-18-002 (2019).

[30] CMS collaboration, Precision luminosity measurement in proton-proton collisions at $\sqrt{s}=13$ TeV in 2015 and 2016 at CMS, Eur. Phys. J. C 81 (2021) 800 [CMS-LUM-17-003] [CERN-EP-2021-033] [arXiv:2104.01927] [INSPIRE].

[31] H.B. Prosper and L. Lyons eds., Proceedings of the PHYSTAT 2011 Workshop on Statistical Issues Related to Discovery Claims in Search Experiments and Unfolding, CERN, Geneva, Switzerland, 17-20 January 2011, in CERN Yellow Reports: Conference Proceedings, CERN, Geneva Switzerland (2011) [CERN-2011-006].

[32] ATLAS, CMS collaborations and LHC Higgs Combination Group, Procedure for the LHC Higgs boson search combination in Summer 2011, CMS-NOTE-2011-005 [ATL-PHYS-PUB-2011-11] (2011).

[33] CMS collaboration, Precise determination of the mass of the Higgs boson and tests of compatibility of its couplings with the standard model predictions using proton collisions at 7 and 8 TeV, Eur. Phys. J. C 75 (2015) 212 [arXiv: 1412.8662] [InSPIRE].

[34] G. Cowan, K. Cranmer, E. Gross and O. Vitells, Asymptotic formulae for likelihood-based tests of new physics, Eur. Phys. J. C 71 (2011) 1554 [Erratum ibid. 73 (2013) 2501] [arXiv: 1007.1727] [INSPIRE].

[35] O.J.P. Éboli, M.C. Gonzalez-Garcia and J.K. Mizukoshi, $p p \rightarrow j j e^{ \pm} \mu^{ \pm} \nu \nu$ and jje $\mu^{\mp} \nu \nu$ at $\mathcal{O}\left(\alpha_{\mathrm{em}}^{6}\right)$ and $\mathcal{O}\left(\alpha_{\mathrm{em}}^{4} \alpha_{s}^{2}\right)$ for the study of the quartic electroweak gauge boson vertex at CERN LHC, Phys. Rev. D 74 (2006) 073005 [hep-ph/0606118] [InSPIRE].

[36] CMS collaboration, Observation of electroweak production of $W \gamma$ with two jets in proton-proton collisions at $\sqrt{s}=13$ TeV, Phys. Lett. B 811 (2020) 135988 [arXiv: 2008.10521] [INSPIRE].

[37] ATLAS collaboration, Studies of $Z \gamma$ production in association with a high-mass dijet system in pp collisions at $\sqrt{s}=8 \mathrm{TeV}$ with the ATLAS detector, JHEP 07 (2017) 107 [arXiv: 1705.01966] [INSPIRE].

[38] CMS collaboration, Measurement of the cross section for electroweak production of a $Z$ boson, a photon and two jets in proton-proton collisions at $\sqrt{s}=13 \mathrm{TeV}$ and constraints on anomalous quartic couplings, JHEP 06 (2020) 076 [arXiv:2002.09902] [INSPIRE].

[39] CMS collaboration, Evidence for electroweak production of four charged leptons and two jets in proton-proton collisions at $\sqrt{s}=13$ TeV, Phys. Lett. B 812 (2021) 135992 [arXiv:2008.07013] [INSPIRE]. 


\section{The CMS collaboration}

\section{Yerevan Physics Institute, Yerevan, Armenia}

A. Tumasyan

\section{Institut für Hochenergiephysik, Wien, Austria}

W. Adam, J.W. Andrejkovic, T. Bergauer, S. Chatterjee, M. Dragicevic, A. Es-

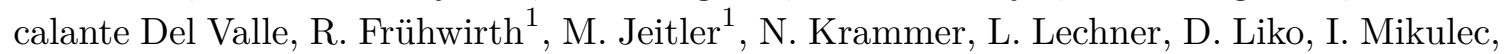
P. Paulitsch, F.M. Pitters, J. Schieck ${ }^{1}$, R. Schöfbeck, M. Spanring, S. Templ, W. Waltenberger, C.-E. Wulz ${ }^{1}$

Institute for Nuclear Problems, Minsk, Belarus

V. Chekhovsky, A. Litomin, V. Makarenko

\section{Universiteit Antwerpen, Antwerpen, Belgium}

M.R. Darwish ${ }^{2}$, E.A. De Wolf, X. Janssen, T. Kello ${ }^{3}$, A. Lelek, H. Rejeb Sfar, P. Van Mechelen, S. Van Putte, N. Van Remortel

\section{Vrije Universiteit Brussel, Brussel, Belgium}

F. Blekman, E.S. Bols, J. D'Hondt, J. De Clercq, M. Delcourt, H. El Faham, S. Lowette, S. Moortgat, A. Morton, D. Müller, A.R. Sahasransu, S. Tavernier, W. Van Doninck, P. Van Mulders

\section{Université Libre de Bruxelles, Bruxelles, Belgium}

D. Beghin, B. Bilin, B. Clerbaux, G. De Lentdecker, L. Favart, A. Grebenyuk, A.K. Kalsi, K. Lee, M. Mahdavikhorrami, I. Makarenko, L. Moureaux, L. Pétré, A. Popov, N. Postiau, E. Starling, L. Thomas, M. Vanden Bemden, C. Vander Velde, P. Vanlaer, D. Vannerom, L. Wezenbeek

\section{Ghent University, Ghent, Belgium}

T. Cornelis, D. Dobur, J. Knolle, L. Lambrecht, G. Mestdach, M. Niedziela, C. Roskas, A. Samalan, K. Skovpen, T.T. Tran, M. Tytgat, W. Verbeke, B. Vermassen, M. Vit

\section{Université Catholique de Louvain, Louvain-la-Neuve, Belgium}

A. Bethani, G. Bruno, F. Bury, C. Caputo, P. David, C. Delaere, I.S. Donertas, A. Giammanco, K. Jaffel, V. Lemaitre, K. Mondal, J. Prisciandaro, A. Taliercio, M. Teklishyn, P. Vischia, S. Wertz, S. Wuyckens

\section{Centro Brasileiro de Pesquisas Fisicas, Rio de Janeiro, Brazil}

G.A. Alves, C. Hensel, A. Moraes

\section{Universidade do Estado do Rio de Janeiro, Rio de Janeiro, Brazil}

W.L. Aldá Júnior, M. Alves Gallo Pereira, M. Barroso Ferreira Filho, H. Brandao Malbouisson, W. Carvalho, J. Chinellato ${ }^{4}$, E.M. Da Costa, G.G. Da Silveira ${ }^{5}$, D. De Jesus Damiao, S. Fonseca De Souza, D. Matos Figueiredo, C. Mora Herrera, K. Mota Amarilo, L. Mundim, H. Nogima, P. Rebello Teles, A. Santoro, S.M. Silva Do Amaral, A. Sznajder, M. Thiel, F. Torres Da Silva De Araujo, A. Vilela Pereira 
Universidade Estadual Paulista ${ }^{a}$, Universidade Federal do $\mathrm{ABC}^{b}$, São Paulo, Brazil

C.A. Bernardes ${ }^{a, a}$, L. Calligaris ${ }^{a}$, T.R. Fernandez Perez Tomei $^{a}$, E.M. Gregores ${ }^{a, b}$, D.S. Lemos ${ }^{a}$, P.G. Mercadante ${ }^{a, b}$, S.F. Novaes ${ }^{a}$, Sandra S. Padula ${ }^{a}$

Institute for Nuclear Research and Nuclear Energy, Bulgarian Academy of Sciences, Sofia, Bulgaria

A. Aleksandrov, G. Antchev, R. Hadjiiska, P. Iaydjiev, M. Misheva, M. Rodozov,

M. Shopova, G. Sultanov

University of Sofia, Sofia, Bulgaria

A. Dimitrov, T. Ivanov, L. Litov, B. Pavlov, P. Petkov, A. Petrov

Beihang University, Beijing, China

T. Cheng, W. Fang ${ }^{3}$, Q. Guo, T. Javaid ${ }^{6}$, M. Mittal, H. Wang, L. Yuan

Department of Physics, Tsinghua University, Beijing, China

M. Ahmad, G. Bauer, C. Dozen ${ }^{7}$, Z. Hu, J. Martins ${ }^{8}$, Y. Wang, K. Yi ${ }^{9,10}$

Institute of High Energy Physics, Beijing, China

E. Chapon, G.M. Chen ${ }^{6}$, H.S. Chen ${ }^{6}$, M. Chen, F. Iemmi, A. Kapoor, D. Leggat, H. Liao, Z.-A. Liu ${ }^{6}$, V. Milosevic, F. Monti, R. Sharma, J. Tao, J. Thomas-Wilsker, J. Wang, H. Zhang, S. Zhang ${ }^{6}$, J. Zhao

State Key Laboratory of Nuclear Physics and Technology, Peking University, Beijing, China

A. Agapitos, Y. Ban, C. Chen, Q. Huang, A. Levin, Q. Li, X. Lyu, Y. Mao, S.J. Qian, D. Wang, Q. Wang, J. Xiao

\section{Sun Yat-Sen University, Guangzhou, China}

M. Lu, Z. You

Institute of Modern Physics and Key Laboratory of Nuclear Physics and Ionbeam Application (MOE) — Fudan University, Shanghai, China

X. $\mathrm{Gao}^{3}, \mathrm{H}$. Okawa

Zhejiang University, Hangzhou, China

Z. Lin, M. Xiao

Universidad de Los Andes, Bogota, Colombia

C. Avila, A. Cabrera, C. Florez, J. Fraga, A. Sarkar, M.A. Segura Delgado

Universidad de Antioquia, Medellin, Colombia

J. Mejia Guisao, F. Ramirez, J.D. Ruiz Alvarez, C.A. Salazar González

University of Split, Faculty of Electrical Engineering, Mechanical Engineering and Naval Architecture, Split, Croatia

D. Giljanovic, N. Godinovic, D. Lelas, I. Puljak 
University of Split, Faculty of Science, Split, Croatia

Z. Antunovic, M. Kovac, T. Sculac

Institute Rudjer Boskovic, Zagreb, Croatia

V. Brigljevic, D. Ferencek, D. Majumder, M. Roguljic, A. Starodumov ${ }^{11}$, T. Susa

University of Cyprus, Nicosia, Cyprus

A. Attikis, E. Erodotou, A. Ioannou, G. Kole, M. Kolosova, S. Konstantinou, J. Mousa,

C. Nicolaou, F. Ptochos, P.A. Razis, H. Rykaczewski, H. Saka

Charles University, Prague, Czech Republic

M. Finger ${ }^{12}$, M. Finger Jr. ${ }^{12}$, A. Kveton

Escuela Politecnica Nacional, Quito, Ecuador

E. Ayala

Universidad San Francisco de Quito, Quito, Ecuador

E. Carrera Jarrin

Academy of Scientific Research and Technology of the Arab Republic of Egypt, Egyptian Network of High Energy Physics, Cairo, Egypt

A.A. Abdelalim ${ }^{13,14}$, S. Abu Zeid ${ }^{15}$

Center for High Energy Physics (CHEP-FU), Fayoum University, El-Fayoum, Egypt

M.A. Mahmoud, Y. Mohammed

National Institute of Chemical Physics and Biophysics, Tallinn, Estonia

S. Bhowmik, A. Carvalho Antunes De Oliveira, R.K. Dewanjee, K. Ehataht, M. Kadastik,

C. Nielsen, J. Pata, M. Raidal, L. Tani, C. Veelken

Department of Physics, University of Helsinki, Helsinki, Finland

P. Eerola, L. Forthomme, H. Kirschenmann, K. Osterberg, M. Voutilainen

Helsinki Institute of Physics, Helsinki, Finland

S. Bharthuar, E. Brücken, F. Garcia, J. Havukainen, M.S. Kim, R. Kinnunen, T. Lampén,

K. Lassila-Perini, S. Lehti, T. Lindén, M. Lotti, L. Martikainen, J. Ott, H. Siikonen,

E. Tuominen, J. Tuominiemi

Lappeenranta University of Technology, Lappeenranta, Finland

P. Luukka, H. Petrow, T. Tuuva

IRFU, CEA, Université Paris-Saclay, Gif-sur-Yvette, France

C. Amendola, M. Besancon, F. Couderc, M. Dejardin, D. Denegri, J.L. Faure, F. Ferri,

S. Ganjour, A. Givernaud, P. Gras, G. Hamel de Monchenault, P. Jarry, B. Lenzi, E. Locci,

J. Malcles, J. Rander, A. Rosowsky, M.Ö. Sahin, A. Savoy-Navarro ${ }^{16}$, M. Titov, G.B. Yu

Laboratoire Leprince-Ringuet, CNRS/IN2P3, Ecole Polytechnique, Institut Polytechnique de Paris, Palaiseau, France

S. Ahuja, F. Beaudette, M. Bonanomi, A. Buchot Perraguin, P. Busson, A. Cappati, C. Charlot, O. Davignon, B. Diab, G. Falmagne, S. Ghosh, R. Granier de Cassagnac, 
A. Hakimi, I. Kucher, M. Nguyen, C. Ochando, P. Paganini, J. Rembser, R. Salerno, J.B. Sauvan, Y. Sirois, A. Zabi, A. Zghiche

Université de Strasbourg, CNRS, IPHC UMR 7178, Strasbourg, France J.-L. Agram ${ }^{17}$, J. Andrea, D. Apparu, D. Bloch, G. Bourgatte, J.-M. Brom, E.C. Chabert, C. Collard, D. Darej, J.-C. Fontaine ${ }^{17}$, U. Goerlach, C. Grimault, A.-C. Le Bihan, E. Nibigira, P. Van Hove

Institut de Physique des 2 Infinis de Lyon (IP2I ), Villeurbanne, France

E. Asilar, S. Beauceron, C. Bernet, G. Boudoul, C. Camen, A. Carle, N. Chanon, D. Contardo, P. Depasse, H. El Mamouni, J. Fay, S. Gascon, M. Gouzevitch, B. Ille, Sa. Jain, I.B. Laktineh, H. Lattaud, A. Lesauvage, M. Lethuillier, L. Mirabito, S. Perries, K. Shchablo, V. Sordini, L. Torterotot, G. Touquet, M. Vander Donckt, S. Viret

\section{Georgian Technical University, Tbilisi, Georgia}

I. Lomidze, T. Toriashvili ${ }^{18}, \mathrm{Z}$. Tsamalaidze ${ }^{12}$

RWTH Aachen University, I. Physikalisches Institut, Aachen, Germany

L. Feld, K. Klein, M. Lipinski, D. Meuser, A. Pauls, M.P. Rauch, N. Röwert, J. Schulz, M. Teroerde

RWTH Aachen University, III. Physikalisches Institut A, Aachen, Germany

D. Eliseev, M. Erdmann, P. Fackeldey, B. Fischer, S. Ghosh, T. Hebbeker, K. Hoepfner, F. Ivone, H. Keller, L. Mastrolorenzo, M. Merschmeyer, A. Meyer, G. Mocellin, S. Mondal, S. Mukherjee, D. Noll, A. Novak, T. Pook, A. Pozdnyakov, Y. Rath, H. Reithler, J. Roemer, A. Schmidt, S.C. Schuler, A. Sharma, S. Wiedenbeck, S. Zaleski

RWTH Aachen University, III. Physikalisches Institut B, Aachen, Germany

C. Dziwok, G. Flügge, W. Haj Ahmad ${ }^{19}$, O. Hlushchenko, T. Kress, A. Nowack, C. Pistone, O. Pooth, D. Roy, H. Sert, A. Stahl ${ }^{20}$, T. Ziemons

\section{Deutsches Elektronen-Synchrotron, Hamburg, Germany}

H. Aarup Petersen, M. Aldaya Martin, P. Asmuss, I. Babounikau, S. Baxter, O. Behnke, A. Bermúdez Martínez, S. Bhattacharya, A.A. Bin Anuar, K. Borras ${ }^{21}$, V. Botta, D. Brunner, A. Campbell, A. Cardini, C. Cheng, S. Consuegra Rodríguez, G. Correia Silva, V. Danilov, L. Didukh, G. Eckerlin, D. Eckstein, L.I. Estevez Banos, O. Filatov, E. Gallo ${ }^{22}$, A. Geiser, A. Giraldi, A. Grohsjean, M. Guthoff, A. Jafari ${ }^{23}$, N.Z. Jomhari, H. Jung, A. Kasem ${ }^{21}$, M. Kasemann, H. Kaveh, C. Kleinwort, D. Krücker, W. Lange, J. Lidrych, K. Lipka, W. Lohmann ${ }^{24}$, R. Mankel, I.-A. Melzer-Pellmann, J. Metwally, A.B. Meyer, M. Meyer, J. Mnich, A. Mussgiller, Y. Otarid, D. Pérez Adán, D. Pitzl, A. Raspereza, B. Ribeiro Lopes, J. Rübenach, A. Saggio, A. Saibel, M. Savitskyi, M. Scham, V. Scheurer, C. Schwanenberger ${ }^{22}$, A. Singh, R.E. Sosa Ricardo, D. Stafford, N. Tonon, O. Turkot, M. Van De Klundert, R. Walsh, D. Walter, Y. Wen, K. Wichmann, L. Wiens, C. Wissing, S. Wuchterl

\section{University of Hamburg, Hamburg, Germany}

R. Aggleton, S. Bein, L. Benato, A. Benecke, P. Connor, K. De Leo, M. Eich, F. Feindt, A. Fröhlich, C. Garbers, E. Garutti, P. Gunnellini, J. Haller, A. Hinzmann, G. Kasieczka, 
R. Klanner, R. Kogler, T. Kramer, V. Kutzner, J. Lange, T. Lange, A. Lobanov, A. Malara, A. Nigamova, K.J. Pena Rodriguez, O. Rieger, P. Schleper, M. Schröder, J. Schwandt, D. Schwarz, J. Sonneveld, H. Stadie, G. Steinbrück, A. Tews, B. Vormwald, I. Zoi

Karlsruher Institut fuer Technologie, Karlsruhe, Germany

J. Bechtel, T. Berger, E. Butz, R. Caspart, T. Chwalek, W. De Boer ${ }^{\dagger}$, A. Dierlamm, A. Droll, K. El Morabit, N. Faltermann, M. Giffels, J.o. Gosewisch, A. Gottmann, F. Hartmann ${ }^{20}$, C. Heidecker, U. Husemann, I. Katkov ${ }^{25}$, P. Keicher, R. Koppenhöfer, S. Maier, M. Metzler, S. Mitra, Th. Müller, M. Neukum, A. Nürnberg, G. Quast, K. Rabbertz, J. Rauser, D. Savoiu, M. Schnepf, D. Seith, I. Shvetsov, H.J. Simonis, R. Ulrich, J. Van Der Linden, R.F. Von Cube, M. Wassmer, M. Weber, S. Wieland, R. Wolf, S. Wozniewski, S. Wunsch

Institute of Nuclear and Particle Physics (INPP), NCSR Demokritos, Aghia Paraskevi, Greece

G. Anagnostou, P. Asenov, G. Daskalakis, T. Geralis, A. Kyriakis, D. Loukas, A. Stakia

National and Kapodistrian University of Athens, Athens, Greece

M. Diamantopoulou, D. Karasavvas, G. Karathanasis, P. Kontaxakis, C.K. Koraka, A. Manousakis-Katsikakis, A. Panagiotou, I. Papavergou, N. Saoulidou, K. Theofilatos, E. Tziaferi, K. Vellidis, E. Vourliotis

National Technical University of Athens, Athens, Greece

G. Bakas, K. Kousouris, I. Papakrivopoulos, G. Tsipolitis, A. Zacharopoulou

University of Ioánnina, Ioánnina, Greece

I. Evangelou, C. Foudas, P. Gianneios, P. Katsoulis, P. Kokkas, N. Manthos, I. Papadopoulos, J. Strologas

MTA-ELTE Lendület CMS Particle and Nuclear Physics Group, Eötvös Loránd University, Budapest, Hungary

M. Csanad, K. Farkas, M.M.A. Gadallah ${ }^{26}$, S. Lökös ${ }^{27}$, P. Major, K. Mandal, A. Mehta, G. Pasztor, A.J. Rádl, O. Surányi, G.I. Veres

Wigner Research Centre for Physics, Budapest, Hungary

M. Bartók ${ }^{28}$, G. Bencze, C. Hajdu, D. Horvath ${ }^{29}$, F. Sikler, V. Veszpremi, G. Vesztergombi ${ }^{\dagger}$

Institute of Nuclear Research ATOMKI, Debrecen, Hungary

S. Czellar, J. Karancsi ${ }^{28}$, J. Molnar, Z. Szillasi, D. Teyssier

Institute of Physics, University of Debrecen, Debrecen, Hungary

P. Raics, Z.L. Trocsanyi ${ }^{30}$, B. Ujvari

Karoly Robert Campus, MATE Institute of Technology

T. Csorgo ${ }^{31}$, F. Nemes ${ }^{31}$, T. Novak

Indian Institute of Science (IISc), Bangalore, India

J.R. Komaragiri, D. Kumar, L. Panwar, P.C. Tiwari 
National Institute of Science Education and Research, HBNI, Bhubaneswar, India

S. Bahinipati ${ }^{32}$, D. Dash, C. Kar, P. Mal, T. Mishra, V.K. Muraleedharan Nair Bindhu ${ }^{33}$, A. Nayak ${ }^{33}$, P. Saha, N. Sur, S.K. Swain, D. Vats ${ }^{33}$

Panjab University, Chandigarh, India

S. Bansal, S.B. Beri, V. Bhatnagar, G. Chaudhary, S. Chauhan, N. Dhingra ${ }^{34}$, R. Gupta, A. Kaur, M. Kaur, S. Kaur, P. Kumari, M. Meena, K. Sandeep, J.B. Singh, A.K. Virdi

University of Delhi, Delhi, India

A. Ahmed, A. Bhardwaj, B.C. Choudhary, M. Gola, S. Keshri, A. Kumar, M. Naimuddin, P. Priyanka, K. Ranjan, A. Shah

Saha Institute of Nuclear Physics, HBNI, Kolkata, India

M. Bharti ${ }^{35}$, R. Bhattacharya, S. Bhattacharya, D. Bhowmik, S. Dutta, S. Dutta, B. Gomber ${ }^{36}$, M. Maity ${ }^{37}$, S. Nandan, P. Palit, P.K. Rout, G. Saha, B. Sahu, S. Sarkar, M. Sharan, B. Singh ${ }^{35}$, S. Thakur ${ }^{35}$

Indian Institute of Technology Madras, Madras, India

P.K. Behera, S.C. Behera, P. Kalbhor, A. Muhammad, R. Pradhan, P.R. Pujahari, A. Sharma, A.K. Sikdar

Bhabha Atomic Research Centre, Mumbai, India

D. Dutta, V. Jha, V. Kumar, D.K. Mishra, K. Naskar ${ }^{38}$, P.K. Netrakanti, L.M. Pant, P. Shukla

Tata Institute of Fundamental Research-A, Mumbai, India

T. Aziz, S. Dugad, M. Kumar, U. Sarkar

Tata Institute of Fundamental Research-B, Mumbai, India

S. Banerjee, R. Chudasama, M. Guchait, S. Karmakar, S. Kumar, G. Majumder, K. Mazumdar, S. Mukherjee

Indian Institute of Science Education and Research (IISER), Pune, India

K. Alpana, S. Dube, B. Kansal, S. Pandey, A. Rane, A. Rastogi, S. Sharma

Department of Physics, Isfahan University of Technology, Isfahan, Iran

H. Bakhshiansohi ${ }^{39}$, M. Zeinali ${ }^{40}$

Institute for Research in Fundamental Sciences (IPM), Tehran, Iran

S. Chenarani ${ }^{41}$, S.M. Etesami, M. Khakzad, M. Mohammadi Najafabadi

University College Dublin, Dublin, Ireland

M. Grunewald

INFN Sezione di Bari ${ }^{a}$, Università di Bari ${ }^{b}$, Politecnico di Bari ${ }^{c}$, Bari, Italy

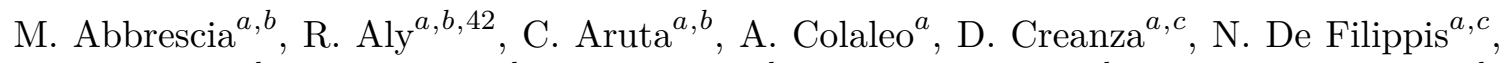
M. De Palma ${ }^{a, b}$, A. Di Florio ${ }^{a, b}$, A. Di Pilato ${ }^{a, b}$, W. Elmetenawee ${ }^{a, b}$, L. Fiore $^{a}$, A. Gelmi $^{a, b}$,

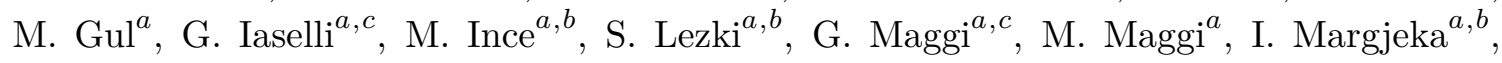


V. Mastrapasqua ${ }^{a, b}$, J.A. Merlin ${ }^{a}$, S. My ${ }^{a, b}$, S. Nuzzo ${ }^{a, b}$, A. Pellecchia $^{a, b}$, A. Pompili ${ }^{a, b}$,

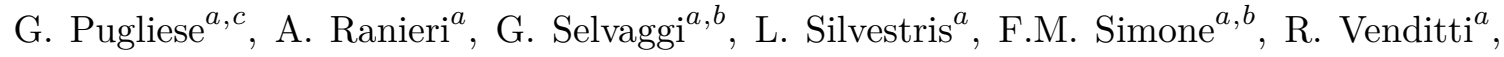
P. Verwilligen ${ }^{a}$

INFN Sezione di Bologna ${ }^{a}$, Università di Bologna ${ }^{b}$, Bologna, Italy

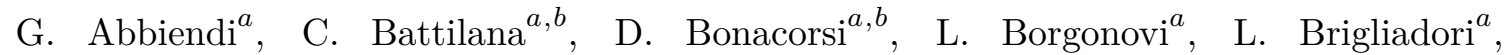
R. Campanini ${ }^{a, b}$, P. Capiluppi ${ }^{a, b}$, A. Castro ${ }^{a, b}$, F.R. Cavallo ${ }^{a}$, M. Cuffiani $^{a, b}$, G.M. Dallavalle ${ }^{a}$, T. Diotalevi ${ }^{a, b}$, F. Fabbri ${ }^{a}$, A. Fanfani ${ }^{a, b}$, P. Giacomelli $^{a}$, L. Giommi $^{a, b}{ }^{,}$ C. Grandi ${ }^{a}$, L. Guiducci ${ }^{a, b}$, S. Lo Meo ${ }^{a, 43}$, L. Lunerti ${ }^{a, b}$, S. Marcellini ${ }^{a}$, G. Masetti ${ }^{a}$, F.L. Navarria ${ }^{a, b}$, A. Perrotta ${ }^{a}$, F. Primavera ${ }^{a, b}$, A.M. Rossi ${ }^{a, b}$, T. Rovelli ${ }^{a, b}$, G.P. Siroli ${ }^{a, b}$

INFN Sezione di Catania ${ }^{a}$, Università di Catania ${ }^{b}$, Catania, Italy

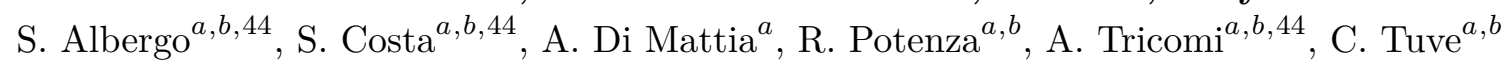

INFN Sezione di Firenze ${ }^{a}$, Università di Firenze ${ }^{b}$, Firenze, Italy

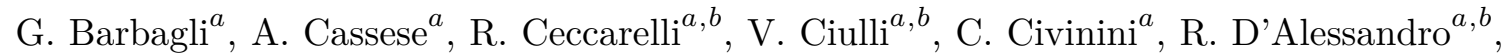

E. Focardi ${ }^{a, b}$, G. Latino ${ }^{a, b}$, P. Lenzi ${ }^{a, b}$, M. Lizzo ${ }^{a, b}$, M. Meschini ${ }^{a}$, S. Paoletti ${ }^{a}$, R. Seidita ${ }^{a, b}$, G. Sguazzoni ${ }^{a}$, L. Viliani ${ }^{a}$

INFN Laboratori Nazionali di Frascati, Frascati, Italy

L. Benussi, S. Bianco, D. Piccolo

INFN Sezione di Genova ${ }^{a}$, Università di Genova ${ }^{b}$, Genova, Italy

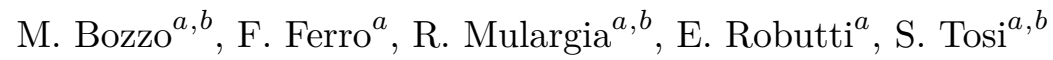

INFN Sezione di Milano-Bicocca $^{a}$, Università di Milano-Bicocca ${ }^{b}$, Milano, Italy

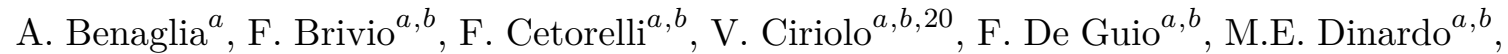
P. Dini ${ }^{a}$, S. Gennai ${ }^{a}$, A. Ghezzi ${ }^{a, b}$, P. Govoni ${ }^{a}, b$, L. Guzzi $^{a}, b$, M. Malberti $^{a}$, S. Malvezzi $^{a}$, A. Massironi ${ }^{a}$, D. Menasce ${ }^{a}$, L. Moroni ${ }^{a}$, M. Paganoni ${ }^{a, b}$, D. Pedrini ${ }^{a}$, S. Ragazzi $^{a, b}$, N. Redaelli ${ }^{a}$, T. Tabarelli de Fatis ${ }^{a, b}$, D. Valsecchi ${ }^{a, b, 20}$, D. Zuolo ${ }^{a, b}$

INFN Sezione di Napoli ${ }^{a}$, Università di Napoli 'Federico II' ${ }^{b}$, Napoli, Italy, Università della Basilicata $^{c}$, Potenza, Italy, Università G. Marconi ${ }^{d}$, Roma, Italy

S. Buontempo ${ }^{a}$, F. Carnevali ${ }^{a, b}$, N. Cavallo ${ }^{a, c}$, A. De Iorio $^{a, b}$, F. Fabozzi $^{a, c}$,

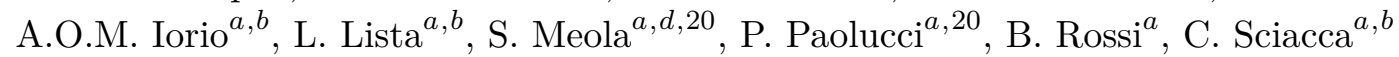

INFN Sezione di Padova ${ }^{a}$, Università di Padova ${ }^{b}$, Padova, Italy, Università di Trento ${ }^{c}$, Trento, Italy

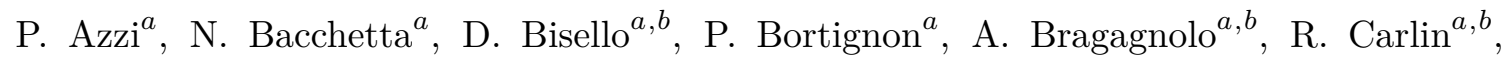

P. Checchia ${ }^{a}$, T. Dorigo ${ }^{a}$, U. Dosselli ${ }^{a}$, F. Gasparini ${ }^{a, b}$, U. Gasparini ${ }^{a, b}$, S.Y. Hoh ${ }^{a, b}$,

L. Layer $^{a, 45}$, M. Margoni ${ }^{a, b}$, A.T. Meneguzzo ${ }^{a, b}$, J. Pazzini ${ }^{a, b}$, M. Presilla ${ }^{a, b}$,

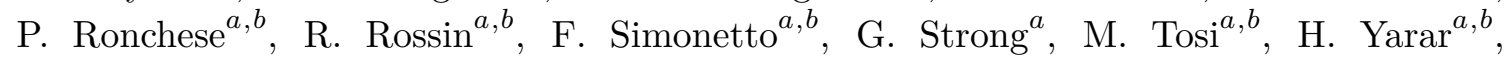
M. Zanetti ${ }^{a, b}$, P. Zotto ${ }^{a, b}$, A. Zucchetta ${ }^{a, b}$, G. Zumerle ${ }^{a, b}$

INFN Sezione di Pavia ${ }^{a}$, Università di Pavia ${ }^{b}$, Pavia, Italy

C. Aimè $\grave{a}^{a, b}$ A. Braghieri ${ }^{a}$, S. Calzaferri ${ }^{a, b}$, D. Fiorina ${ }^{a, b}$, P. Montagna ${ }^{a, b}$, S.P. Ratti ${ }^{a, b}$,

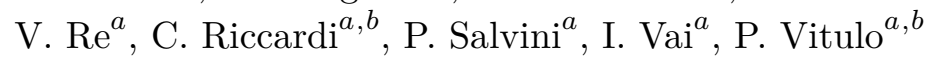


INFN Sezione di Perugia ${ }^{a}$, Università di Perugia ${ }^{b}$, Perugia, Italy G.M. Bilei ${ }^{a}$, D. Ciangottini ${ }^{a, b}$, L. Fanò ${ }^{a, b}$, P. Lariccia ${ }^{a, b}$, M. Magherini $^{b}$, G. Mantovani $^{a, b}{ }^{b}$ V. Mariani ${ }^{a, b}$, M. Menichelli ${ }^{a}$, F. Moscatelli $^{a}$, A. Piccinelli $^{a, b}$, A. Rossi $^{a, b}$, A. Santocchia $^{a, b}$, D. Spiga ${ }^{a}$, T. Tedeschi ${ }^{a, b}$

INFN Sezione di Pisa ${ }^{a}$, Università di Pisa ${ }^{b}$, Scuola Normale Superiore di Pisa ${ }^{c}$, Pisa Italy, Università di Siena ${ }^{d}$, Siena, Italy

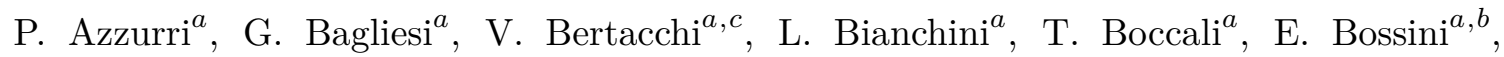
R. Castaldi ${ }^{a}$, M.A. Ciocci ${ }^{a, b}$, R. Dell'Orso ${ }^{a}$, M.R. Di Domenico ${ }^{a, d}$, S. Donato $^{a}$, A. Giassi $^{a}$, M.T. Grippo ${ }^{a}$, F. Ligabue ${ }^{a, c}$, E. Manca ${ }^{a, c}$, G. Mandorli ${ }^{a, c}$, A. Messineo ${ }^{a, b}$, F. Palla $^{a}$, S. Parolia ${ }^{a, b}$, G. Ramirez-Sanchez ${ }^{a, c}$, A. Rizzi ${ }^{a, b}$, G. Rolandi ${ }^{a, c}$, S. Roy Chowdhury ${ }^{a, c}$,

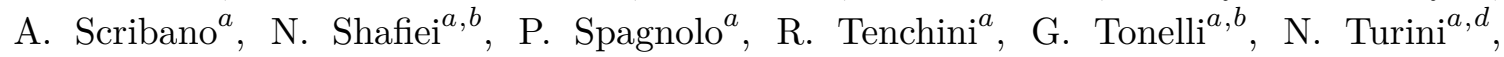
A. Venturi ${ }^{a}$, P.G. Verdini ${ }^{a}$

INFN Sezione di Roma ${ }^{a}$, Sapienza Università di Roma ${ }^{b}$, Rome, Italy

M. Campana ${ }^{a, b}$, F. Cavallari ${ }^{a}$, M. Cipriani ${ }^{a}, b$, D. Del Re ${ }^{a, b}$, E. Di Marco ${ }^{a}$, M. Diemoz $^{a}$,

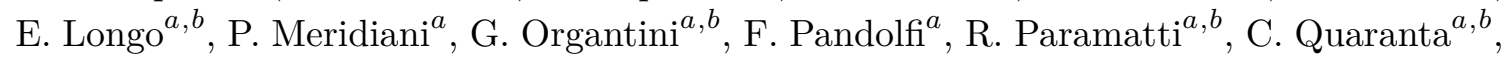

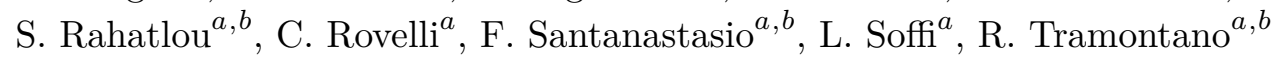

INFN Sezione di Torino ${ }^{a}$, Università di Torino ${ }^{b}$, Torino, Italy, Università del Piemonte Orientale ${ }^{c}$, Novara, Italy

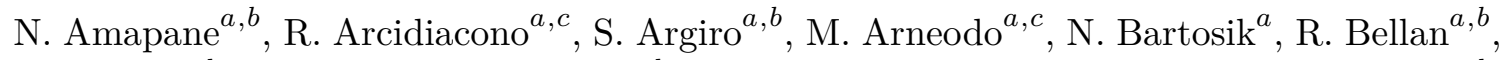
A. Bellora ${ }^{a, b}$, J. Berenguer Antequera $^{a, b}$, C. Biino ${ }^{a}$, N. Cartiglia $^{a}$, S. Cometti $^{a}$, M. Costa $^{a, b}$, R. Covarelli ${ }^{a, b}$, N. Demaria ${ }^{a}$, B. Kiani ${ }^{a, b}$, F. Legger ${ }^{a}$, C. Mariotti $^{a}$, S. Maselli ${ }^{a}$, E. Migliore $^{a, b}$, E. Monteil ${ }^{a, b}$, M. Monteno ${ }^{a}$, M.M. Obertino ${ }^{a, b}$, G. Ortona $^{a}$, L. Pacher ${ }^{a, b}$, N. Pastrone ${ }^{a}$, M. Pelliccioni ${ }^{a}$, G.L. Pinna Angioni ${ }^{a, b}$, M. Ruspa ${ }^{a, c}$, R. Salvatico $^{a, b}$, K. Shchelina ${ }^{a, b}$, F. Siviero ${ }^{a, b}$, V. Sola ${ }^{a}$, A. Solano ${ }^{a, b}$, D. Soldi ${ }^{a, b}$, A. Staiano ${ }^{a}$, M. Tornago ${ }^{a, b}$, D. Trocino ${ }^{a, b}$, A. Vagnerini

INFN Sezione di Trieste ${ }^{a}$, Università di Trieste ${ }^{b}$, Trieste, Italy

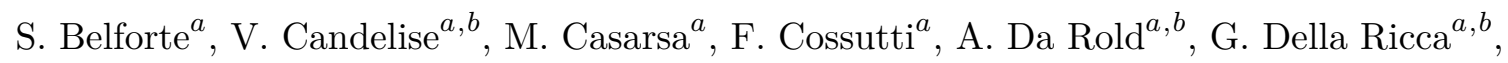
$\mathrm{G}$ Sorrentino ${ }^{a, b}, \mathrm{~F}$. Vazzoler ${ }^{a, b}$

Kyungpook National University, Daegu, Korea

S. Dogra, C. Huh, B. Kim, D.H. Kim, G.N. Kim, J. Kim, J. Lee, S.W. Lee, C.S. Moon, Y.D. Oh, S.I. Pak, B.C. Radburn-Smith, S. Sekmen, Y.C. Yang

Chonnam National University, Institute for Universe and Elementary Particles, Kwangju, Korea

H. Kim, D.H. Moon

Hanyang University, Seoul, Korea

B. Francois, T.J. Kim, J. Park

Korea University, Seoul, Korea

S. Cho, S. Choi, Y. Go, B. Hong, K. Lee, K.S. Lee, J. Lim, J. Park, S.K. Park, J. Yoo 
Kyung Hee University, Department of Physics, Seoul, Republic of Korea

J. Goh, A. Gurtu

Sejong University, Seoul, Korea

H.S. Kim, Y. Kim

Seoul National University, Seoul, Korea

J. Almond, J.H. Bhyun, J. Choi, S. Jeon, J. Kim, J.S. Kim, S. Ko, H. Kwon, H. Lee, S. Lee, B.H. Oh, M. Oh, S.B. Oh, H. Seo, U.K. Yang, I. Yoon

University of Seoul, Seoul, Korea

W. Jang, D. Jeon, D.Y. Kang, Y. Kang, J.H. Kim, S. Kim, B. Ko, J.S.H. Lee, Y. Lee, I.C. Park, Y. Roh, M.S. Ryu, D. Song, I.J. Watson, S. Yang

Yonsei University, Department of Physics, Seoul, Korea

S. Ha, H.D. Yoo

Sungkyunkwan University, Suwon, Korea

Y. Jeong, H. Lee, Y. Lee, I. Yu

College of Engineering and Technology, American University of the Middle East (AUM), Egaila, Kuwait

T. Beyrouthy, Y. Maghrbi

Riga Technical University, Riga, Latvia

V. Veckalns ${ }^{46}$

Vilnius University, Vilnius, Lithuania

M. Ambrozas, A. Juodagalvis, A. Rinkevicius, G. Tamulaitis, A. Vaitkevicius

National Centre for Particle Physics, Universiti Malaya, Kuala Lumpur, Malaysia

N. Bin Norjoharuddeen, W.A.T. Wan Abdullah, M.N. Yusli, Z. Zolkapli

Universidad de Sonora (UNISON), Hermosillo, Mexico

J.F. Benitez, A. Castaneda Hernandez, M. León Coello, J.A. Murillo Quijada, A. Sehrawat, L. Valencia Palomo

Centro de Investigacion y de Estudios Avanzados del IPN, Mexico City, Mexico G. Ayala, H. Castilla-Valdez, I. Heredia-De La Cruz ${ }^{47}$, R. Lopez-Fernandez, C.A. Mondragon Herrera, D.A. Perez Navarro, A. Sanchez-Hernandez

Universidad Iberoamericana, Mexico City, Mexico

S. Carrillo Moreno, C. Oropeza Barrera, M. Ramirez-Garcia, F. Vazquez Valencia

Benemerita Universidad Autonoma de Puebla, Puebla, Mexico

I. Pedraza, H.A. Salazar Ibarguen, C. Uribe Estrada

University of Montenegro, Podgorica, Montenegro

J. Mijuskovic $^{48}$, N. Raicevic 
University of Auckland, Auckland, New Zealand

D. Krofcheck

University of Canterbury, Christchurch, New Zealand

S. Bheesette, P.H. Butler

National Centre for Physics, Quaid-I-Azam University, Islamabad, Pakistan

A. Ahmad, M.I. Asghar, A. Awais, M.I.M. Awan, H.R. Hoorani, W.A. Khan, M.A. Shah, M. Shoaib, M. Waqas

AGH University of Science and Technology Faculty of Computer Science, Electronics and Telecommunications, Krakow, Poland

V. Avati, L. Grzanka, M. Malawski

National Centre for Nuclear Research, Swierk, Poland

H. Bialkowska, M. Bluj, B. Boimska, M. Górski, M. Kazana, M. Szleper, P. Zalewski

Institute of Experimental Physics, Faculty of Physics, University of Warsaw, Warsaw, Poland

K. Bunkowski, K. Doroba, A. Kalinowski, M. Konecki, J. Krolikowski, M. Walczak

Laboratório de Instrumentação e Física Experimental de Partículas, Lisboa, Portugal

M. Araujo, P. Bargassa, D. Bastos, A. Boletti, P. Faccioli, M. Gallinaro, J. Hollar, N. Leonardo, T. Niknejad, M. Pisano, J. Seixas, O. Toldaiev, J. Varela

Joint Institute for Nuclear Research, Dubna, Russia

S. Afanasiev, D. Budkouski, I. Golutvin, I. Gorbunov, V. Karjavine, V. Korenkov, A. Lanev, A. Malakhov, V. Matveev ${ }^{49,50}$, V. Palichik, V. Perelygin, M. Savina, D. Seitova, V. Shalaev, S. Shmatov, S. Shulha, V. Smirnov, O. Teryaev, N. Voytishin, B.S. Yuldashev ${ }^{51}$, A. Zarubin, I. Zhizhin

Petersburg Nuclear Physics Institute, Gatchina (St. Petersburg), Russia

G. Gavrilov, V. Golovtcov, Y. Ivanov, V. Kim ${ }^{52}$, E. Kuznetsova ${ }^{53}$, V. Murzin, V. Oreshkin, I. Smirnov, D. Sosnov, V. Sulimov, L. Uvarov, S. Volkov, A. Vorobyev

Institute for Nuclear Research, Moscow, Russia

Yu. Andreev, A. Dermenev, S. Gninenko, N. Golubev, A. Karneyeu, D. Kirpichnikov, M. Kirsanov, N. Krasnikov, A. Pashenkov, G. Pivovarov, D. Tlisov ${ }^{\dagger}$, A. Toropin

Institute for Theoretical and Experimental Physics named by A.I. Alikhanov of NRC 'Kurchatov Institute', Moscow, Russia

V. Epshteyn, V. Gavrilov, N. Lychkovskaya, A. Nikitenko ${ }^{54}$, V. Popov, A. Spiridonov, A. Stepennov, M. Toms, E. Vlasov, A. Zhokin

Moscow Institute of Physics and Technology, Moscow, Russia

T. Aushev 
National Research Nuclear University 'Moscow Engineering Physics Institute' (MEPhI), Moscow, Russia

M. Chadeeva ${ }^{55}$, A. Oskin, P. Parygin, E. Popova, V. Rusinov

P.N. Lebedev Physical Institute, Moscow, Russia

V. Andreev, M. Azarkin, I. Dremin, M. Kirakosyan, A. Terkulov

Skobeltsyn Institute of Nuclear Physics, Lomonosov Moscow State University, Moscow, Russia

A. Belyaev, E. Boos, M. Dubinin ${ }^{56}$, L. Dudko, A. Ershov, A. Gribushin, V. Klyukhin, O. Kodolova, I. Lokhtin, S. Obraztsov, S. Petrushanko, V. Savrin, A. Snigirev

Novosibirsk State University (NSU), Novosibirsk, Russia

V. Blinov ${ }^{57}$, T. Dimova ${ }^{57}$, L. Kardapoltsev ${ }^{57}$, A. Kozyrev ${ }^{57}$, I. Ovtin ${ }^{57}$, Y. Skovpen ${ }^{57}$

Institute for High Energy Physics of National Research Centre 'Kurchatov Institute', Protvino, Russia

I. Azhgirey, I. Bayshev, D. Elumakhov, V. Kachanov, D. Konstantinov, P. Mandrik, V. Petrov, R. Ryutin, S. Slabospitskii, A. Sobol, S. Troshin, N. Tyurin, A. Uzunian, A. Volkov

National Research Tomsk Polytechnic University, Tomsk, Russia

A. Babaev, V. Okhotnikov

Tomsk State University, Tomsk, Russia

V. Borchsh, V. Ivanchenko, E. Tcherniaev

University of Belgrade: Faculty of Physics and VINCA Institute of Nuclear Sciences, Belgrade, Serbia

P. Adzic ${ }^{58}$, M. Dordevic, P. Milenovic, J. Milosevic

Centro de Investigaciones Energéticas Medioambientales y Tecnológicas (CIEMAT), Madrid, Spain

M. Aguilar-Benitez, J. Alcaraz Maestre, A. Álvarez Fernández, I. Bachiller, M. Barrio Luna, Cristina F. Bedoya, C.A. Carrillo Montoya, M. Cepeda, M. Cerrada, N. Colino, B. De La Cruz, A. Delgado Peris, J.P. Fernández Ramos, J. Flix, M.C. Fouz, O. Gonzalez Lopez, S. Goy Lopez, J.M. Hernandez, M.I. Josa, J. León Holgado, D. Moran, Á. Navarro Tobar, A. Pérez-Calero Yzquierdo, J. Puerta Pelayo, I. Redondo, L. Romero, S. Sánchez Navas, L. Urda Gómez, C. Willmott

Universidad Autónoma de Madrid, Madrid, Spain

J.F. de Trocóniz, R. Reyes-Almanza

Universidad de Oviedo, Instituto Universitario de Ciencias y Tecnologías Espaciales de Asturias (ICTEA), Oviedo, Spain

B. Alvarez Gonzalez, J. Cuevas, C. Erice, J. Fernandez Menendez, S. Folgueras, I. Gonzalez Caballero, E. Palencia Cortezon, C. Ramón Álvarez, J. Ripoll Sau, V. Rodríguez Bouza, A. Trapote, N. Trevisani 
Instituto de Física de Cantabria (IFCA), CSIC-Universidad de Cantabria, Santander, Spain

J.A. Brochero Cifuentes, I.J. Cabrillo, A. Calderon, J. Duarte Campderros, M. Fernandez, C. Fernandez Madrazo, P.J. Fernández Manteca, A. García Alonso, G. Gomez, C. Martinez Rivero, P. Martinez Ruiz del Arbol, F. Matorras, P. Matorras Cuevas, J. Piedra Gomez, C. Prieels, T. Rodrigo, A. Ruiz-Jimeno, L. Scodellaro, I. Vila, J.M. Vizan Garcia

\section{University of Colombo, Colombo, Sri Lanka}

M.K. Jayananda, B. Kailasapathy ${ }^{59}$, D.U.J. Sonnadara, D.D.C. Wickramarathna

\section{University of Ruhuna, Department of Physics, Matara, Sri Lanka}

W.G.D. Dharmaratna, K. Liyanage, N. Perera, N. Wickramage

\section{CERN, European Organization for Nuclear Research, Geneva, Switzerland}

T.K. Aarrestad, D. Abbaneo, J. Alimena, E. Auffray, G. Auzinger, J. Baechler, P. Baillon ${ }^{\dagger}$, D. Barney, J. Bendavid, M. Bianco, A. Bocci, T. Camporesi, M. Capeans Garrido, G. Cerminara, S.S. Chhibra, L. Cristella, D. d'Enterria, A. Dabrowski, N. Daci, A. David, A. De Roeck, M.M. Defranchis, M. Deile, M. Dobson, M. Dünser, N. Dupont, A. ElliottPeisert, N. Emriskova, F. Fallavollita ${ }^{60}$, D. Fasanella, S. Fiorendi, A. Florent, G. Franzoni, W. Funk, S. Giani, D. Gigi, K. Gill, F. Glege, L. Gouskos, M. Haranko, J. Hegeman, Y. Iiyama, V. Innocente, T. James, P. Janot, J. Kaspar, J. Kieseler, M. Komm, N. Kratochwil, C. Lange, S. Laurila, P. Lecoq, K. Long, C. Lourenço, L. Malgeri, S. Mallios, M. Mannelli, A.C. Marini, F. Meijers, S. Mersi, E. Meschi, F. Moortgat, M. Mulders, S. Orfanelli, L. Orsini, F. Pantaleo, L. Pape, E. Perez, M. Peruzzi, A. Petrilli, G. Petrucciani, A. Pfeiffer, M. Pierini, D. Piparo, M. Pitt, H. Qu, T. Quast, D. Rabady, A. Racz, G. Reales Gutiérrez, M. Rieger, M. Rovere, H. Sakulin, J. Salfeld-Nebgen, S. Scarfi, C. Schäfer, C. Schwick, M. Selvaggi, A. Sharma, P. Silva, W. Snoeys, P. Sphicas ${ }^{61}$, S. Summers, V.R. Tavolaro, D. Treille, A. Tsirou, G.P. Van Onsem, M. Verzetti, J. Wanczyk ${ }^{62}$, K.A. Wozniak, W.D. Zeuner

\section{Paul Scherrer Institut, Villigen, Switzerland}

L. Caminada ${ }^{63}$, A. Ebrahimi, W. Erdmann, R. Horisberger, Q. Ingram, H.C. Kaestli, D. Kotlinski, U. Langenegger, M. Missiroli, T. Rohe

ETH Zurich - Institute for Particle Physics and Astrophysics (IPA), Zurich, Switzerland

K. Androsov ${ }^{62}$, M. Backhaus, P. Berger, A. Calandri, N. Chernyavskaya, A. De Cosa, G. Dissertori, M. Dittmar, M. Donegà, C. Dorfer, F. Eble, T.A. Gómez Espinosa, C. Grab, D. Hits, W. Lustermann, A.-M. Lyon, R.A. Manzoni, C. Martin Perez, M.T. Meinhard, F. Micheli, F. Nessi-Tedaldi, J. Niedziela, F. Pauss, V. Perovic, G. Perrin, S. Pigazzini, M.G. Ratti, M. Reichmann, C. Reissel, T. Reitenspiess, B. Ristic, D. Ruini, D.A. Sanz Becerra, M. Schönenberger, V. Stampf, J. Steggemann ${ }^{62}$, R. Wallny, D.H. Zhu 
Universität Zürich, Zurich, Switzerland

C. Amsler ${ }^{64}$, P. Bärtschi, C. Botta, D. Brzhechko, M.F. Canelli, K. Cormier, A. De Wit, R. Del Burgo, J.K. Heikkilä, M. Huwiler, A. Jofrehei, B. Kilminster, S. Leontsinis, A. Macchiolo, P. Meiring, V.M. Mikuni, U. Molinatti, I. Neutelings, A. Reimers, P. Robmann, S. Sanchez Cruz, K. Schweiger, Y. Takahashi

\section{National Central University, Chung-Li, Taiwan}

C. Adloff ${ }^{65}$, C.M. Kuo, W. Lin, A. Roy, T. Sarkar ${ }^{37}$, S.S. Yu

National Taiwan University (NTU), Taipei, Taiwan

L. Ceard, Y. Chao, K.F. Chen, P.H. Chen, W.-S. Hou, Y.y. Li, R.-S. Lu, E. Paganis,

A. Psallidas, A. Steen, H.y. Wu, E. Yazgan, P.r. Yu

Chulalongkorn University, Faculty of Science, Department of Physics, Bangkok, Thailand

B. Asavapibhop, C. Asawatangtrakuldee, N. Srimanobhas

Çukurova University, Physics Department, Science and Art Faculty, Adana, Turkey

F. Boran, S. Damarseckin ${ }^{66}$, Z.S. Demiroglu, F. Dolek, I. Dumanoglu ${ }^{67}$, E. Eskut, Y. Guler,

E. Gurpinar Guler ${ }^{68}$, I. Hos $^{69}$, C. Isik, O. Kara, A. Kayis Topaksu, U. Kiminsu, G. Onengut,

K. Ozdemir ${ }^{70}$, A. Polatoz, A.E. Simsek, B. Tali ${ }^{71}$, U.G. Tok, S. Turkcapar, I.S. Zorbakir,

C. Zorbilmez

Middle East Technical University, Physics Department, Ankara, Turkey

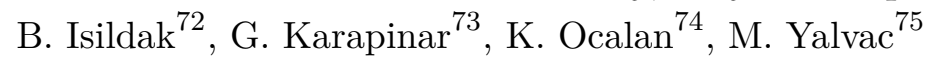

Bogazici University, Istanbul, Turkey

B. Akgun, I.O. Atakisi, E. Gülmez, M. Kaya ${ }^{76}$, O. Kaya ${ }^{77}$, Ö. Özçelik, S. Tekten ${ }^{78}$, E.A. Yetkin ${ }^{79}$

Istanbul Technical University, Istanbul, Turkey

A. Cakir, K. Cankocak ${ }^{67}$, Y. Komurcu, S. Sen ${ }^{80}$

Istanbul University, Istanbul, Turkey

S. Cerci ${ }^{71}$, B. Kaynak, S. Ozkorucuklu, D. Sunar Cerci ${ }^{71}$

Institute for Scintillation Materials of National Academy of Science of Ukraine, Kharkov, Ukraine

B. Grynyov

National Scientific Center, Kharkov Institute of Physics and Technology, Kharkov, Ukraine

L. Levchuk

University of Bristol, Bristol, U.K.

D. Anthony, E. Bhal, S. Bologna, J.J. Brooke, A. Bundock, E. Clement, D. Cussans,

H. Flacher, J. Goldstein, G.P. Heath, H.F. Heath, L. Kreczko, B. Krikler, S. Paramesvaran,

S. Seif El Nasr-Storey, V.J. Smith, N. Stylianou ${ }^{81}$, R. White 
Rutherford Appleton Laboratory, Didcot, U.K.

K.W. Bell, A. Belyaev ${ }^{82}$, C. Brew, R.M. Brown, D.J.A. Cockerill, K.V. Ellis, K. Harder, S. Harper, J. Linacre, K. Manolopoulos, D.M. Newbold, E. Olaiya, D. Petyt, T. Reis, T. Schuh, C.H. Shepherd-Themistocleous, I.R. Tomalin, T. Williams

Imperial College, London, U.K.

R. Bainbridge, P. Bloch, S. Bonomally, J. Borg, S. Breeze, O. Buchmuller, V. Cepaitis, G.S. Chahal ${ }^{83}$, D. Colling, P. Dauncey, G. Davies, M. Della Negra, S. Fayer, G. Fedi, G. Hall, M.H. Hassanshahi, G. Iles, J. Langford, L. Lyons, A.-M. Magnan, S. Malik, A. Martelli, J. Nash ${ }^{84}$, M. Pesaresi, D.M. Raymond, A. Richards, A. Rose, E. Scott, C. Seez, A. Shtipliyski, A. Tapper, K. Uchida, T. Virdee ${ }^{20}$, N. Wardle, S.N. Webb, D. Winterbottom, A.G. Zecchinelli

Brunel University, Uxbridge, U.K.

K. Coldham, J.E. Cole, A. Khan, P. Kyberd, I.D. Reid, L. Teodorescu, S. Zahid

Baylor University, Waco, U.S.A.

S. Abdullin, A. Brinkerhoff, B. Caraway, J. Dittmann, K. Hatakeyama, A.R. Kanuganti, B. McMaster, N. Pastika, S. Sawant, C. Sutantawibul, J. Wilson

Catholic University of America, Washington, DC, U.S.A.

R. Bartek, A. Dominguez, R. Uniyal, A.M. Vargas Hernandez

The University of Alabama, Tuscaloosa, U.S.A.

A. Buccilli, S.I. Cooper, D. Di Croce, S.V. Gleyzer, C. Henderson, C.U. Perez, P. Rumerio ${ }^{85}$, C. West

Boston University, Boston, U.S.A.

A. Akpinar, A. Albert, D. Arcaro, C. Cosby, Z. Demiragli, E. Fontanesi, D. Gastler, J. Rohlf, K. Salyer, D. Sperka, D. Spitzbart, I. Suarez, A. Tsatsos, S. Yuan, D. Zou

Brown University, Providence, U.S.A.

G. Benelli, B. Burkle, X. Coubez ${ }^{21}$, D. Cutts, M. Hadley, U. Heintz, J.M. Hogan ${ }^{86}$, G. Landsberg, K.T. Lau, M. Lukasik, J. Luo, M. Narain, S. Sagir ${ }^{87}$, E. Usai, W.Y. Wong, X. Yan, D. Yu, W. Zhang

University of California, Davis, Davis, U.S.A.

J. Bonilla, C. Brainerd, R. Breedon, M. Calderon De La Barca Sanchez, M. Chertok, J. Conway, P.T. Cox, R. Erbacher, G. Haza, F. Jensen, O. Kukral, R. Lander, M. Mulhearn, D. Pellett, B. Regnery, D. Taylor, Y. Yao, F. Zhang

University of California, Los Angeles, U.S.A.

M. Bachtis, R. Cousins, A. Datta, D. Hamilton, J. Hauser, M. Ignatenko, M.A. Iqbal, T. Lam, N. Mccoll, W.A. Nash, S. Regnard, D. Saltzberg, B. Stone, V. Valuev

University of California, Riverside, Riverside, U.S.A.

K. Burt, Y. Chen, R. Clare, J.W. Gary, M. Gordon, G. Hanson, G. Karapostoli, O.R. Long, N. Manganelli, M. Olmedo Negrete, W. Si, S. Wimpenny, Y. Zhang 
University of California, San Diego, La Jolla, U.S.A.

J.G. Branson, P. Chang, S. Cittolin, S. Cooperstein, N. Deelen, J. Duarte, R. Gerosa, L. Giannini, D. Gilbert, J. Guiang, R. Kansal, V. Krutelyov, R. Lee, J. Letts, M. Masciovecchio, S. May, M. Pieri, B.V. Sathia Narayanan, V. Sharma, M. Tadel, A. Vartak, F. Würthwein, Y. Xiang, A. Yagil

University of California, Santa Barbara - Department of Physics, Santa Barbara, U.S.A.

N. Amin, C. Campagnari, M. Citron, A. Dorsett, V. Dutta, J. Incandela, M. Kilpatrick, J. Kim, B. Marsh, H. Mei, M. Oshiro, M. Quinnan, J. Richman, U. Sarica, D. Stuart, S. Wang

California Institute of Technology, Pasadena, U.S.A.

A. Bornheim, O. Cerri, I. Dutta, J.M. Lawhorn, N. Lu, J. Mao, H.B. Newman, J. Ngadiuba, T.Q. Nguyen, M. Spiropulu, J.R. Vlimant, C. Wang, S. Xie, Z. Zhang, R.Y. Zhu

Carnegie Mellon University, Pittsburgh, U.S.A.

J. Alison, S. An, M.B. Andrews, P. Bryant, T. Ferguson, A. Harilal, C. Liu, T. Mudholkar, M. Paulini, A. Sanchez

University of Colorado Boulder, Boulder, U.S.A.

J.P. Cumalat, W.T. Ford, A. Hassani, E. MacDonald, R. Patel, A. Perloff, C. Savard, K. Stenson, K.A. Ulmer, S.R. Wagner

Cornell University, Ithaca, U.S.A.

J. Alexander, Y. Cheng, D.J. Cranshaw, S. Hogan, J. Monroy, J.R. Patterson, D. Quach, J. Reichert, A. Ryd, W. Sun, J. Thom, P. Wittich, R. Zou

Fermi National Accelerator Laboratory, Batavia, U.S.A.

M. Albrow, M. Alyari, G. Apollinari, A. Apresyan, A. Apyan, S. Banerjee, L.A.T. Bauerdick, D. Berry, J. Berryhill, P.C. Bhat, K. Burkett, J.N. Butler, A. Canepa, G.B. Cerati, H.W.K. Cheung, F. Chlebana, M. Cremonesi, K.F. Di Petrillo, V.D. Elvira, Y. Feng, J. Freeman, Z. Gecse, L. Gray, D. Green, S. Grünendahl, O. Gutsche, R.M. Harris, R. Heller, T.C. Herwig, J. Hirschauer, B. Jayatilaka, S. Jindariani, M. Johnson, U. Joshi, T. Klijnsma, B. Klima, K.H.M. Kwok, S. Lammel, D. Lincoln, R. Lipton, T. Liu, C. Madrid, K. Maeshima, C. Mantilla, D. Mason, P. McBride, P. Merkel, S. Mrenna, S. Nahn, V. O'Dell, V. Papadimitriou, K. Pedro, C. Pena ${ }^{56}$, O. Prokofyev, F. Ravera, A. Reinsvold Hall, L. Ristori, B. Schneider, E. Sexton-Kennedy, N. Smith, A. Soha, W.J. Spalding, L. Spiegel, S. Stoynev, J. Strait, L. Taylor, S. Tkaczyk, N.V. Tran, L. Uplegger, E.W. Vaandering, H.A. Weber

\section{University of Florida, Gainesville, U.S.A.}

D. Acosta, P. Avery, D. Bourilkov, L. Cadamuro, V. Cherepanov, F. Errico, R.D. Field, D. Guerrero, B.M. Joshi, M. Kim, E. Koenig, J. Konigsberg, A. Korytov, K.H. Lo, K. Matchev, N. Menendez, G. Mitselmakher, A. Muthirakalayil Madhu, N. Rawal, D. Rosenzweig, S. Rosenzweig, K. Shi, J. Sturdy, J. Wang, E. Yigitbasi, X. Zuo 
Florida State University, Tallahassee, U.S.A.

T. Adams, A. Askew, D. Diaz, R. Habibullah, V. Hagopian, K.F. Johnson, R. Khurana,

T. Kolberg, G. Martinez, H. Prosper, C. Schiber, R. Yohay, J. Zhang

Florida Institute of Technology, Melbourne, U.S.A.

M.M. Baarmand, S. Butalla, T. Elkafrawy ${ }^{15}$, M. Hohlmann, R. Kumar Verma, D. Noonan, M. Rahmani, M. Saunders, F. Yumiceva

University of Illinois at Chicago (UIC), Chicago, U.S.A.

M.R. Adams, H. Becerril Gonzalez, R. Cavanaugh, X. Chen, S. Dittmer, O. Evdokimov, C.E. Gerber, D.A. Hangal, D.J. Hofman, A.H. Merrit, C. Mills, G. Oh, T. Roy, S. Rudrabhatla, M.B. Tonjes, N. Varelas, J. Viinikainen, X. Wang, Z. Wu, Z. Ye

The University of Iowa, Iowa City, U.S.A.

M. Alhusseini, K. Dilsiz ${ }^{88}$, R.P. Gandrajula, O.K. Köseyan, J.-P. Merlo, A. Mestvirishvili ${ }^{89}$, J. Nachtman, H. Ogul ${ }^{90}$, Y. Onel, A. Penzo, C. Snyder, E. Tiras ${ }^{91}$

Johns Hopkins University, Baltimore, U.S.A.

O. Amram, B. Blumenfeld, L. Corcodilos, J. Davis, M. Eminizer, A.V. Gritsan, S. Kyriacou, P. Maksimovic, J. Roskes, M. Swartz, T.Á. Vámi

The University of Kansas, Lawrence, U.S.A.

J. Anguiano, C. Baldenegro Barrera, P. Baringer, A. Bean, A. Bylinkin, T. Isidori, S. Khalil, J. King, G. Krintiras, A. Kropivnitskaya, C. Lindsey, N. Minafra, M. Murray, C. Rogan, C. Royon, S. Sanders, E. Schmitz, C. Smith, J.D. Tapia Takaki, Q. Wang, J. Williams, G. Wilson

\section{Kansas State University, Manhattan, U.S.A.}

S. Duric, A. Ivanov, K. Kaadze, D. Kim, Y. Maravin, T. Mitchell, A. Modak, K. Nam

Lawrence Livermore National Laboratory, Livermore, U.S.A.

F. Rebassoo, D. Wright

University of Maryland, College Park, U.S.A.

E. Adams, A. Baden, O. Baron, A. Belloni, S.C. Eno, N.J. Hadley, S. Jabeen, R.G. Kellogg,

T. Koeth, A.C. Mignerey, S. Nabili, M. Seidel, A. Skuja, L. Wang, K. Wong

Massachusetts Institute of Technology, Cambridge, U.S.A.

D. Abercrombie, G. Andreassi, R. Bi, S. Brandt, W. Busza, I.A. Cali, Y. Chen, M. D'Alfonso, J. Eysermans, G. Gomez Ceballos, M. Goncharov, P. Harris, M. Hu, M. Klute, D. Kovalskyi, J. Krupa, Y.-J. Lee, B. Maier, C. Mironov, C. Paus, D. Rankin, C. Roland, G. Roland, Z. Shi, G.S.F. Stephans, K. Tatar, J. Wang, Z. Wang, B. Wyslouch

University of Minnesota, Minneapolis, U.S.A.

R.M. Chatterjee, A. Evans, P. Hansen, J. Hiltbrand, Sh. Jain, M. Krohn, Y. Kubota, J. Mans, M. Revering, R. Rusack, R. Saradhy, N. Schroeder, N. Strobbe, M.A. Wadud 
University of Nebraska-Lincoln, Lincoln, U.S.A.

K. Bloom, M. Bryson, S. Chauhan, D.R. Claes, C. Fangmeier, L. Finco, F. Golf, J.R. González Fernández, C. Joo, I. Kravchenko, M. Musich, I. Reed, J.E. Siado, G.R. Snow ${ }^{\dagger}$, W. Tabb, F. Yan

State University of New York at Buffalo, Buffalo, U.S.A.

G. Agarwal, H. Bandyopadhyay, L. Hay, I. Iashvili, A. Kharchilava, C. McLean, D. Nguyen, J. Pekkanen, S. Rappoccio, A. Williams

Northeastern University, Boston, U.S.A.

G. Alverson, E. Barberis, C. Freer, Y. Haddad, A. Hortiangtham, J. Li, G. Madigan, B. Marzocchi, D.M. Morse, V. Nguyen, T. Orimoto, A. Parker, L. Skinnari, A. TishelmanCharny, T. Wamorkar, B. Wang, A. Wisecarver, D. Wood

Northwestern University, Evanston, U.S.A.

S. Bhattacharya, J. Bueghly, Z. Chen, A. Gilbert, T. Gunter, K.A. Hahn, N. Odell, M.H. Schmitt, M. Velasco

University of Notre Dame, Notre Dame, U.S.A.

R. Band, R. Bucci, A. Das, N. Dev, R. Goldouzian, M. Hildreth, K. Hurtado Anampa, C. Jessop, K. Lannon, N. Loukas, N. Marinelli, I. Mcalister, T. McCauley, F. Meng, K. Mohrman, Y. Musienko ${ }^{49}$, R. Ruchti, P. Siddireddy, M. Wayne, A. Wightman, M. Wolf, M. Zarucki, L. Zygala

The Ohio State University, Columbus, U.S.A.

B. Bylsma, B. Cardwell, L.S. Durkin, B. Francis, C. Hill, M. Nunez Ornelas, K. Wei, B.L. Winer, B.R. Yates

Princeton University, Princeton, U.S.A.

F.M. Addesa, B. Bonham, P. Das, G. Dezoort, P. Elmer, A. Frankenthal, B. Greenberg, N. Haubrich, S. Higginbotham, A. Kalogeropoulos, G. Kopp, S. Kwan, D. Lange, M.T. Lucchini, D. Marlow, K. Mei, I. Ojalvo, J. Olsen, C. Palmer, D. Stickland, C. Tully

University of Puerto Rico, Mayaguez, U.S.A.

S. Malik, S. Norberg

Purdue University, West Lafayette, U.S.A.

A.S. Bakshi, V.E. Barnes, R. Chawla, S. Das, L. Gutay, M. Jones, A.W. Jung, S. Karmarkar, M. Liu, G. Negro, N. Neumeister, G. Paspalaki, C.C. Peng, S. Piperov, A. Purohit, J.F. Schulte, M. Stojanovic ${ }^{16}$, J. Thieman, F. Wang, R. Xiao, W. Xie

Purdue University Northwest, Hammond, U.S.A.

J. Dolen, N. Parashar

Rice University, Houston, U.S.A.

A. Baty, M. Decaro, S. Dildick, K.M. Ecklund, S. Freed, P. Gardner, F.J.M. Geurts, A. Kumar, W. Li, B.P. Padley, R. Redjimi, W. Shi, A.G. Stahl Leiton, S. Yang, L. Zhang, Y. Zhang 
University of Rochester, Rochester, U.S.A.

A. Bodek, P. de Barbaro, R. Demina, J.L. Dulemba, C. Fallon, T. Ferbel, M. Galanti, A. Garcia-Bellido, O. Hindrichs, A. Khukhunaishvili, E. Ranken, R. Taus

Rutgers, The State University of New Jersey, Piscataway, U.S.A.

B. Chiarito, J.P. Chou, A. Gandrakota, Y. Gershtein, E. Halkiadakis, A. Hart, M. Heindl, E. Hughes, S. Kaplan, O. Karacheban ${ }^{24}$, I. Laflotte, A. Lath, R. Montalvo, K. Nash, M. Osherson, S. Salur, S. Schnetzer, S. Somalwar, R. Stone, S.A. Thayil, S. Thomas, H. Wang

University of Tennessee, Knoxville, U.S.A.

H. Acharya, A.G. Delannoy, S. Spanier

Texas A\&M University, College Station, U.S.A.

O. Bouhali ${ }^{92}$, M. Dalchenko, A. Delgado, R. Eusebi, J. Gilmore, T. Huang, T. Kamon ${ }^{93}$, H. Kim, S. Luo, S. Malhotra, R. Mueller, D. Overton, D. Rathjens, A. Safonov

Texas Tech University, Lubbock, U.S.A.

N. Akchurin, J. Damgov, V. Hegde, S. Kunori, K. Lamichhane, S.W. Lee, T. Mengke, S. Muthumuni, T. Peltola, I. Volobouev, Z. Wang, A. Whitbeck

Vanderbilt University, Nashville, U.S.A.

E. Appelt, S. Greene, A. Gurrola, W. Johns, A. Melo, H. Ni, K. Padeken, F. Romeo, P. Sheldon, S. Tuo, J. Velkovska

University of Virginia, Charlottesville, U.S.A.

M.W. Arenton, B. Cox, G. Cummings, J. Hakala, R. Hirosky, M. Joyce, A. Ledovskoy, A. Li, C. Neu, B. Tannenwald, S. White, E. Wolfe

Wayne State University, Detroit, U.S.A.

N. Poudyal

University of Wisconsin - Madison, Madison, WI, U.S.A.

K. Black, T. Bose, J. Buchanan, C. Caillol, S. Dasu, I. De Bruyn, P. Everaerts, F. Fienga, C. Galloni, H. He, M. Herndon, A. Hervé, U. Hussain, A. Lanaro, A. Loeliger, R. Loveless, J. Madhusudanan Sreekala, A. Mallampalli, A. Mohammadi, D. Pinna, A. Savin, V. Shang, V. Sharma, W.H. Smith, D. Teague, S. Trembath-Reichert, W. Vetens

$\dagger$ : Deceased

1: Also at TU Wien, Wien, Austria

2: Also at Institute of Basic and Applied Sciences, Faculty of Engineering, Arab Academy for Science, Technology and Maritime Transport, Alexandria, Egypt, Alexandria, Egypt

3: Also at Université Libre de Bruxelles, Bruxelles, Belgium

4: Also at Universidade Estadual de Campinas, Campinas, Brazil

5: Also at Federal University of Rio Grande do Sul, Porto Alegre, Brazil

6: Also at University of Chinese Academy of Sciences, Beijing, China

7: Also at Department of Physics, Tsinghua University, Beijing, China, Beijing, China

8: Also at UFMS, Nova Andradina, Brazil 
9: Also at Nanjing Normal University Department of Physics, Nanjing, China

10: Now at The University of Iowa, Iowa City, U.S.A.

11: Also at Institute for Theoretical and Experimental Physics named by A.I. Alikhanov of NRC 'Kurchatov Institute', Moscow, Russia

12: Also at Joint Institute for Nuclear Research, Dubna, Russia

13: Also at Helwan University, Cairo, Egypt

14: Now at Zewail City of Science and Technology, Zewail, Egypt

15: Also at Ain Shams University, Cairo, Egypt

16: Also at Purdue University, West Lafayette, U.S.A.

17: Also at Université de Haute Alsace, Mulhouse, France

18: Also at Tbilisi State University, Tbilisi, Georgia

19: Also at Erzincan Binali Yildirim University, Erzincan, Turkey

20: Also at CERN, European Organization for Nuclear Research, Geneva, Switzerland

21: Also at RWTH Aachen University, III. Physikalisches Institut A, Aachen, Germany

22: Also at University of Hamburg, Hamburg, Germany

23: Also at Department of Physics, Isfahan University of Technology, Isfahan, Iran, Isfahan, Iran

24: Also at Brandenburg University of Technology, Cottbus, Germany

25: Also at Skobeltsyn Institute of Nuclear Physics, Lomonosov Moscow State University, Moscow, Russia

26: Also at Physics Department, Faculty of Science, Assiut University, Assiut, Egypt

27: Also at Karoly Robert Campus, MATE Institute of Technology, Gyongyos, Hungary

28: Also at Institute of Physics, University of Debrecen, Debrecen, Hungary, Debrecen, Hungary

29: Also at Institute of Nuclear Research ATOMKI, Debrecen, Hungary

30: Also at MTA-ELTE Lendület CMS Particle and Nuclear Physics Group, Eötvös Loránd University, Budapest, Hungary, Budapest, Hungary

31: Also at Wigner Research Centre for Physics, Budapest, Hungary

32: Also at IIT Bhubaneswar, Bhubaneswar, India, Bhubaneswar, India

33: Also at Institute of Physics, Bhubaneswar, India

34: Also at G.H.G. Khalsa College, Punjab, India

35: Also at Shoolini University, Solan, India

36: Also at University of Hyderabad, Hyderabad, India

37: Also at University of Visva-Bharati, Santiniketan, India

38: Also at Indian Institute of Technology (IIT), Mumbai, India

39: Also at Deutsches Elektronen-Synchrotron, Hamburg, Germany

40: Also at Sharif University of Technology, Tehran, Iran

41: Also at Department of Physics, University of Science and Technology of Mazandaran, Behshahr, Iran

42: Now at INFN Sezione di Bari ${ }^{a}$, Università di $\mathrm{Bari}^{b}$, Politecnico di Bari ${ }^{c}$, Bari, Italy

43: Also at Italian National Agency for New Technologies, Energy and Sustainable Economic Development, Bologna, Italy

44: Also at Centro Siciliano di Fisica Nucleare e di Struttura Della Materia, Catania, Italy

45: Also at Università di Napoli 'Federico II', Napoli, Italy

46: Also at Riga Technical University, Riga, Latvia, Riga, Latvia

47: Also at Consejo Nacional de Ciencia y Tecnología, Mexico City, Mexico

48: Also at IRFU, CEA, Université Paris-Saclay, Gif-sur-Yvette, France

49: Also at Institute for Nuclear Research, Moscow, Russia

50: Now at National Research Nuclear University 'Moscow Engineering Physics Institute' (MEPhI), Moscow, Russia 
51: Also at Institute of Nuclear Physics of the Uzbekistan Academy of Sciences, Tashkent, Uzbekistan

52: Also at St. Petersburg State Polytechnical University, St. Petersburg, Russia

53: Also at University of Florida, Gainesville, U.S.A.

54: Also at Imperial College, London, U.K.

55: Also at Moscow Institute of Physics and Technology, Moscow, Russia, Moscow, Russia

56: Also at California Institute of Technology, Pasadena, U.S.A.

57: Also at Budker Institute of Nuclear Physics, Novosibirsk, Russia

58: Also at Faculty of Physics, University of Belgrade, Belgrade, Serbia

59: Also at Trincomalee Campus, Eastern University, Sri Lanka, Nilaveli, Sri Lanka

60: Also at INFN Sezione di Pavia ${ }^{a}$, Università di Pavia ${ }^{b}$, Pavia, Italy, Pavia, Italy

61: Also at National and Kapodistrian University of Athens, Athens, Greece

62: Also at Ecole Polytechnique Fédérale Lausanne, Lausanne, Switzerland

63: Also at Universität Zürich, Zurich, Switzerland

64: Also at Stefan Meyer Institute for Subatomic Physics, Vienna, Austria, Vienna, Austria

65: Also at Laboratoire d'Annecy-le-Vieux de Physique des Particules, IN2P3-CNRS, Annecyle-Vieux, France

66: Also at Şirnak University, Sirnak, Turkey

67: Also at Near East University, Research Center of Experimental Health Science, Nicosia, Turkey

68: Also at Konya Technical University, Konya, Turkey

69: Also at Istanbul University - Cerrahpasa, Faculty of Engineering, Istanbul, Turkey

70: Also at Piri Reis University, Istanbul, Turkey

71: Also at Adiyaman University, Adiyaman, Turkey

72: Also at Ozyegin University, Istanbul, Turkey

73: Also at Izmir Institute of Technology, Izmir, Turkey

74: Also at Necmettin Erbakan University, Konya, Turkey

75: Also at Bozok Universitetesi Rektörlügü, Yozgat, Turkey, Yozgat, Turkey

76: Also at Marmara University, Istanbul, Turkey

77: Also at Milli Savunma University, Istanbul, Turkey

78: Also at Kafkas University, Kars, Turkey

79: Also at Istanbul Bilgi University, Istanbul, Turkey

80: Also at Hacettepe University, Ankara, Turkey

81: Also at Vrije Universiteit Brussel, Brussel, Belgium

82: Also at School of Physics and Astronomy, University of Southampton, Southampton, U.K.

83: Also at IPPP Durham University, Durham, U.K.

84: Also at Monash University, Faculty of Science, Clayton, Australia

85: Also at Università di Torino, Torino, Italy

86: Also at Bethel University, St. Paul, Minneapolis, U.S.A.

87: Also at Karamanoğlu Mehmetbey University, Karaman, Turkey

88: Also at Bingol University, Bingol, Turkey

89: Also at Georgian Technical University, Tbilisi, Georgia

90: Also at Sinop University, Sinop, Turkey

91: Also at Erciyes University, Kayseri, Turkey

92: Also at Texas A\&M University at Qatar, Doha, Qatar

93: Also at Kyungpook National University, Daegu, Korea, Daegu, Korea 